\title{
Revisiting observables in generally covariant theories in the light of gauge fixing methods
}

\author{
J. M. Pons* \\ Departament d'Estructura i Constituents de la Matèria and Institut de Ciències del Cosmos, \\ Universitat de Barcelona, Diagonal 647, 08028 Barcelona, Catalonia, Spain \\ D. C. Salisbury ${ }^{\dagger}$ \\ Max-Planck-Institut für Wissenschaftsgeschichte, Boltzmannstrasse 22, 14195 Berlin, Germany \\ and Department of Physics, Austin College, Sherman, Texas 75090-4440, USA \\ K. A. Sundermeyer \\ Freie Universität Berlin, Fachbereich Physik, Institute for Theoretical Physics, Arnimallee 14, 14195 Berlin, Germany
} (Received 12 June 2009; published 13 October 2009)

\begin{abstract}
We derive for generally covariant theories the generic dependency of observables on the original fields, corresponding to coordinate-dependent gauge fixings. This gauge choice is equivalent to a choice of intrinsically defined coordinates accomplished with the aid of spacetime scalar fields. With our approach we make full contact with, and give a new perspective to, the "evolving constants of motion" program. We are able to directly derive generic properties of observables, especially their dynamics and their Poisson algebra in terms of Dirac brackets, extending earlier results in the literature. We also give a new interpretation of the observables as limits of canonical maps.
\end{abstract}

DOI: 10.1103/PhysRevD.80.084015

PACS numbers: 04.20.Fy, 04.60.Ds

\section{INTRODUCTION}

Theories with gauge symmetries exhibit a mathematically redundant description of the same physical setting. Gauge transformations, defined in the space of field configurations, map solutions of the equations of motion to other solutions that have the same physical contents. Generally covariant theories-like general relativityand Yang-Mills theories are the most relevant examples of this type of theory. The concept of observables for these theories has long been a topic of discussion. We refer for instance to Bergmann's particularly insightful treatment in general relativity [1].

Although there exist different conceptions of observables in theories with gauge symmetries, everyone in the community agrees that these are quantities that are invariant under the respective gauge transformations. Therefore they are sometimes also called "gauge invariants," or simply "invariants." Together with that of "observables," this is the terminology that will be employed here without making any distinction among them. Other language like "complete observables," or "Dirac observables" can be found in the literature. ${ }^{1}$ For generally covariant theories observables are identified with those objects of the theory that are invariant under coordinate transformations. They may be the classical versions of quantum observables, although there is no a priori relation to quantities that

\footnotetext{
*pons@ecm.ub.es

†dsalisbury@austincollege.edu

ksun@gmx.de

${ }^{1}$ One may even distinguish Dirac from Bergmann observables; see [2].
}

can be measured. In canonical versions of generally covariant theories-understood as the first step of canonical quantizations-it is more or less known from the work of Rosenfeld, Dirac, and Bergmann that (1) the Hamiltonian is nothing but a sum of constraints (relations between fields and their canonically conjugate momenta) defining a surface in the phase space, (2) the local symmetries are generated by gauge generators which can be expressed by the constraints, and (3) the observables are those objects which have vanishing Poisson brackets on the constraint surface with the gauge generators.

In the preceding sentences we were simply referring to "the constraints." However, as known from the RosenfeldDirac-Bergmann algorithm, ${ }^{2}$ one must distinguish first and second class constraints as well as various generations of constraints (primary, secondary,...). Whereas the notion of "first/second" class is tied to the Poisson bracket relations among the constraints, the "generation classification" depends on the stage at which a given constraint appears when imposing consistency requirements on the equations of motion (EOM).

It is known from the $(1+3) \mathrm{ADM}$ split that in generally covariant theories the Hamiltonian $\mathcal{H}_{0}$ constraint and the momentum constraints $\mathcal{H}_{a}$ are secondary first-class constraints. The Hamiltonian of the theory is built out of these

\footnotetext{
${ }^{2}$ Although every respectable review of "Constrained Dynamics" mentions the work of L. Rosenfeld it is not well known in the community which results he actually established in 1930, and which later were reestablished especially by Dirac. This will be elaborated from a history-of-science point of view by one of the authors (D. C. Salisbury) in a forthcoming publication in Archive for History of Exact Science. See also [3,4].
} 
constraints, with the possible addition of boundary terms, which may be necessary in order for the Hamiltonian to be a differentiable functional [5]; these terms have no effect on the dynamics, but they can be relevant as regards conserved quantities. There are also primary first-class constraints, namely, the vanishing momenta canonically conjugate to the lapse and shift functions. We define as observables those quantities that have weakly vanishing ${ }^{3}$ Poisson brackets with all first-class constraints. (This definition is not entirely in agreement with the definition of many others who only require weakly vanishing Poisson brackets with the secondary first-class constraints. Both definitions agree if one drops the lapse and shift functions as canonical fields.)

Ultimately the interest in observables is due to the necessity of identifying those quantities that can be predicted from a theory and are subject to measurement. However, in the presence of phase-space constraints this identification is not trivial.

In the case of Yang-Mills theories, with internal-i.e., non spacetime - gauge symmetries, observables are well known, and either local like the trace of the curvature 2form, or nonlocal like the Wilson loop. But, to this date, for generic general relativity no observables are known. Only for some spacetimes with special asymptotic behavior or additional Killing symmetries have observables been constructed; this includes cosmologies and cylindrical waves. In these cases one has been able to construct observables with the help of explicit solutions of the respective field equations. Since the work of Torre [6] one knows that the observables in general are nonlocal, that is, functionals of the original fields and their derivatives. Only recently did Dittrich [7] and Thiemann [8] find a formal expression for these functional invariants in the generic case, and for canonical variables other than lapse and shift. We give an interpretation of Dittrich's and Thiemann's expressions in terms of gauge choices and intrinsic coordinates, where everything is based on the original diffeomorphism symmetry of general relativity. This geometric and physical route toward the observables furthermore permits us to display some interesting properties of the functional invariants. In addition to the authors referred to above, we must mention the work by Lusanna and Pauri [2]. They analyze the notion of the observable, among other issues, from a somewhat different perspective.

This article is written in the spirit of previous work [911] essentially dealing with a deeper understanding of the fate of the diffeomorphism group in the phase-space formulation of generally covariant theories.

In these articles [9-11] it was stressed that:

(i) There is a maximal subgroup of the field-dependent diffeomorphism group that can be realized as ca-

\footnotetext{
${ }^{3}$ In Dirac's notation, "weakly vanishing" means vanishing on the constraint surface.
}

nonical transformations in phase space. This subgroup of "diffeomorphism-induced" transformations can be characterized by asking for Legendre projectability between the configurationvelocity and the phase space of the theory.

(ii) The lapse and the shift functions are not arbitrary Lagrange multipliers in the Dirac Hamiltonian, but are canonical field variables. Otherwise one is not able to realize the full group of four-dimensional diffeomorphisms in phase space. It is true, though, that the dynamics will relate the lapse and the shift variables with the time derivatives of the Lagrange multipliers associated with the primary constraints.

(iii) A specific combination of all first-class constraints constitutes the generator of the diffeomorphisminduced transformations on the original phase space. The Hamiltonian and momentum constraints only generate transformations in a reduced phase space-where the lapse and shift are turned into Lagrange multipliers whereas their canonical momenta (the primary constraints) are eliminatedand they are no longer related to the original fourdimensional diffeomorphism group that included transformations of the coordinate time.

(iv) Time evolution in the phase space of generally covariant theories is distinguished from gauge transformations. On the other hand, projectability issues prevent the gauge group from containing time translations as a subgroup, in the sense that an element of the former group effects the same time translation on all solution trajectories.

(v) The observables may depend on the coordinate time of any observer, which may be quite arbitrary. Thus they are not necessarily constants of motion, although constants of motion can be extracted form them.

(vi) From the perspective of gauge-fixing methods, observables are nothing other than the full set of dynamical variables evaluated in an appropriately chosen intrinsic coordinate system. Equivalently, they may be obtained through a symmetry gauge transformation to the intrinsic coordinate system.

Notice that many of these findings are correlated by simply requiring Legendre projectability. We also point out that some of them undermine folklore in the canonical gravity community. In this paper we will elucidate further the final point, (f), and we will compare with other procedures for the construction of observables that can be found in the literature.

Most of our results are proven to be valid locally. We do not address global issues in this article. Although we could assume for the sake of simplicity that the spacetime is spatially compact without the boundary, this is not really relevant; we do not expect that the local construction of observables is changed by the topology of spacetime or by 
possible extra terms in the Hamiltonian that appear, e.g., due to spatial noncompactness. Of course, as is always the case in canonical gravity we consider only globally hyperbolic spacetimes that admit a $(3+1)$ split.

This article largely expands and gives complete proofs of some results that have been advanced in [12]. In Sec. II we describe two alternative but related procedures for constructing observables. These procedures start from gauge-fixing conditions that explicitly depend on the coordinates in such a way that the gauge choice is equivalent to a choice of intrinsic coordinates. Both procedures amount to sending a point $p$ in the space of field configurations, representing a solution of the field equations, to a point $p_{G}$ where the gauge conditions are fulfilled. An extended Sec. II B describes the first procedure in which observables are constructed through active gauge transformations. This procedure delivers an expression for the transformed field that can be solved explicitly for a set of functions that determine the finite transformation. These so-called "descriptor" functions depend on phase-space variables. Thus we are able to characterize all observables as functional invariants in a generic manner that, to the best of our knowledge, was not previously known. We find that every field-including the lapse and the shift—has an associated observable, as well as any functional combination of fields. Furthermore we are able to state the equations of motion of the invariants-negating claims in the literature that invariants are constants of motion. The second procedure, described in II C, amounts to considering a passive coordinate transformation from $p$ to $p_{G}$. We establish the general relation between the active canonical gauge transformations and the corresponding passive coordinate transformation. This latter transformation is none other than the transformation to intrinsic coordinates taking into account the geometric character of each field.

We should mention here that our usage of "passive" and "active" transformations conforms with that in the community, described in e.g. [13], [14]. That is, passive diffeomorphisms are always understood as coordinate transformations, whereas an active diffeomorphism is a mapping of a manifold to itself that induces pullbacks of the tensor fields of the manifold. Although mathematically distinct-and we will make this distinction also in Appendix A-in many cases they can be made to be two sides of the same coin. The relation of passive and active transformations to each other, to dynamical symmetries of the Einstein field equations, and to gauge transformations is treated in [2].

In Sec. III we examine some properties of the observables that can be derived from their definition, regardless of their specific construction in a given theory. We show that they exhibit a natural dependence on the time coordinatethe time coordinate of the corresponding observer, which, in the case that the observer sits at $p_{G}$, is the intrinsic time and that their Hamiltonian dynamics is in agreement with the dynamics of the gauge-fixed fields at $p_{G}$. We also show that one can extract constants of motion-and Noether generators - with no explicit time dependence out of the invariants. We connect our findings with the notion of "evolving constants of motion" [15-17].

In III B we give a simple geometric proof that the Poisson bracket of the invariants associated with two given fields turns out to be the invariant associated with the Dirac bracket of these fields. This can be understood as giving a symmetry-based interpretation of a proof by Thiemann [8] based on a formal series expansion. Furthermore our proof also includes the lapse and the shift functions.

In IIIC it is shown that the functional invariants can also-rather intriguingly - be understood as limits of canonical maps. This provides an alternative route to the results in Sec. III B.

Our main results are summarized in the concluding section, where we interpret our findings in the light of related work on observables in generally covariant theories.

We devote Appendix A to a more detailed consideration of those points where, as a consequence of our belief in the central role of diffeomorphisms in phase space, our ideas deviate from the opinions of others in the canonical gravity community. In the form of a dialogue, we treat amongst others the issues of gauge transformations and gauge generators, and the different roles of gauge generators and the Hamiltonian. In Appendix B we prove a lemma about appropriately redefined constraints. This proof is a reelaboration of an earlier proof by Thiemann [8], in which he investigated specific linear combinations of the secondary first-class constraints with the property that the new constraints have strongly vanishing Poisson brackets. This technical trick significantly facilitated the explicit construction of observables as well as clarifying relations between Poisson and Dirac brackets of observables.

\section{CONSTRUCTING OBSERVABLES THROUGH COORDINATE-DEPENDENT GAUGE FIXINGS}

We recall [9] that the Legendre-projectable infinitesimal passive coordinate transformations are of the form

$$
\tilde{x}^{\mu}=x^{\mu}-\left[n^{\mu}(x) \xi^{0}\left(x ; g_{a b}\right)-\delta_{a}^{\mu} \xi^{a}\left(x ; g_{a b}\right)\right] \Delta s,
$$

( $\Delta s$ is the infinitesimal parameter associated with the transformation). This decomposition was employed by Bergmann and Komar in [18] in their endeavor to connect the diffeomorphism group of general relativity with the algebra of constraints obtained in its canonical formulation, though they did not recognize that it followed from the requirement of projectability. The generator of the corresponding active canonical phase-space transformation is

$$
G_{\xi}(t)=P_{\mu} \dot{\xi}^{\mu}+\left(\mathcal{H}_{\mu}+N^{\rho} C_{\mu \rho}^{\nu} P_{\nu}\right) \xi^{\mu} .
$$

(Repeated indices signify both a sum over the discrete 
index and a 3-dimensional integration over the spatial coordinates, unless otherwise noted. Different indices correspond to different coordinates.) In this expression $N^{0}:=$ $N$ is the lapse, and the $N^{a}$ are the shift. The $P_{0}$ and $P_{a}$ are their conjugate momenta. They vanish as primary firstclass constraints. The normal to the fixed coordinate time $t=$ constant hypersurface is $n^{\mu}=\left\{N^{-1},-N^{-1} N^{a}\right\}$. The $\mathcal{H}_{\mu}$ are the secondary constraints that result from the preservation under time evolution of the primary constraints $P_{\mu} \approx 0$. The $C_{\mu \rho}^{\nu}$ are the structure coefficients in the algebra of the $\mathcal{H}_{\mu}$ under the Poisson bracket. The descriptors $\xi^{\mu}$ are arbitrary functions of the spacetime coordinates as well as the fields other than the lapse and shift. $\dot{\xi}^{\mu}$ is the time derivative of the descriptors, which includes, in the case when $\xi^{\mu}$ depend on fields, the implicit time dependence for these fields as given by the dynamics. $G_{\xi}(t)$ acts at a single time $t$. In order to produce the full infinitesimal action of an element of the gauge group we need to specify the descriptors $\xi^{\mu}$ for all values of the coordinate $t$.

\section{A. Spacetime scalars as intrinsic coordinates}

The coordinate-dependent gauge-fixing program that we will implement consists in using an appropriate set of four independent scalar field functions $X^{\mu}$ in a given spacetime and then taking them as an "intrinsic" system of coordinates. This implies that the program is only feasible for backgrounds admitting a scalar coordinatization. ${ }^{4}$ One possibility is to employ functions of Weyl scalars that are obtained from the Weyl conformal tensor [20]. This option is also in principle available in nonvaccum spacetimes with material field sources [10]. However, one must be aware that in spacetimes with some Killing symmetries, it is likely that these scalars will not be independent and functions of them could not then play the role of an intrinsic coordinatization. Let us review here a variation of the proof given in [10] that the intrinsic coordinate fields must be spacetime scalars. We interpret a choice of intrinsic coordinates $X^{\mu}(x)$ as a coordinate transformation from the coordinates $x^{\mu}$ to $X^{\mu}$. Suppose that instead of starting with coordinates $x^{\mu}$ we start instead with coordinates $f^{\mu}(x)$ before transforming to the intrinsic coordinate system $X_{f}(f(x))$. Then the demand of invariance under the passage from $x^{\mu}$ to $f^{\mu}(x)$ is the demand that the coordinate transformation from $X^{\mu}$ to $X_{f}^{\mu}$ must be the identity transformation, i.e., invariance is precisely the demand that $X^{\mu}(x)=X_{f}^{\mu}(f(x))$. This is the condition that $X^{\mu}(x)$ is a spacetime scalar.

The idea of using a set of four scalars can be traced back to Einstein's hole argument that spacetime points can only be defined and distinguished by values of physical fields or

\footnotetext{
${ }^{4}$ That our real world admits such coordinatization, at least in a spacetime region, is shown, in particular, in [19].
}

positions of physical objects, [21], and has been stressed in [1,22-25]. One either needs external reference objects like dust [26] or GPS satellites [19], or one identifies internal scalars, like in the Weyl-scalar program initiated by Komar and Bergmann, $[1,20]$.

\section{B. Constructing observables through active gauge transformations}

The gauge-fixing conditions have the form

$$
\chi^{\mu}(x):=x^{\mu}-X^{\mu}(x)=0 .
$$

Notice that this gauge-fixing condition is explicitly coordinate dependent. This coordinate dependence is mandatory and indeed, (cf. $[9,27,28]$ ) one can formally prove that this is the manner in which one guarantees that the resulting dynamical evolution is never "frozen".

For most of our considerations, the arena will be the space $\mathcal{S}$ of on-shell field configurations, i.e., fields obeying the equations of motion. This space is a subset of the much bigger space of general field configurations. An infinitesimal gauge transfomation acts on this bigger space with the ordinary Poisson bracket, and its action can be restricted to $\mathcal{S}$ because the generators of gauge-transformations define an action which is tangent to $\mathcal{S}$. This means that we do not need to know the off-shell extension of the on-shell field configurations for the action of the gauge generators to be well defined on $\mathcal{S}$. A point $p$ in $\mathcal{S}$ is in fact an entire spacetime with the fields-solution of the EOM-described in a particular coordinatization. For practical purposes, though, it will be enough to work in a coordinate patch of a given chart. To every point $p$ there is associated an "observer," or "user," who is using such a coordinatization to describe the fields in spacetime. In particular the time coordinate is a label for a foliation of the spacetime into spacelike hypersurfaces - at least in a region of it. The gauge generators, acting through the Poisson brackets, are used to construct finite gauge transformations, realizing active diffeomorphism-induced transformations at a fixed value of the spacetime coordinates. These gauge transformations define equivalence classes within $\mathcal{S}$, which we call orbits of gauge equivalent spacetimes, or gauge orbits for short. A whole gauge orbit represents a unique physical state, ${ }^{5}$ and its different points correspond to different coordinatizations. One can pass from one coordinatization to another by a passive diffeomorphism. This gauge transformation is however not a dynamical evolution (cf. Appendix $\mathrm{A}^{6}$ ) because whereas a gauge transformation-different from the identity-maps a point $p$ into a different point $p^{\prime}$, the dynamical evolution takes place

\footnotetext{
${ }^{5}$ Possible different understandings - and misunderstandingsof what a physical state is are dealt with in Appendix A

${ }^{6}$ In Appendix A we review the differences between Bergmann's and Dirac's approaches to gauge transformations, and the consequences thereof. The incompleteness of Dirac's view in [29] is analyzed in [30]
} 
entirely within every point $p$ in $\mathcal{S}$-because every point represents a solution of the EOM.

Consider the point $p$ in $\mathcal{S}$ and let the fields at $p$ be $X^{\mu}(x)$, $\Phi^{A}(x)$, where $X^{\mu}(x)$ are the set of selected scalars and $\Phi^{A}(x)$ denote all the remaining fields or field components. There should ${ }^{7}$ exist a finite gauge transformation that moves this point $p$ in the gauge orbit to the unique point, $p_{G}$, that satisfies the gauge-fixing constraints (3). We will also assume a trivial topology for the orbit space-or at least in the region in which we will work-so that a diffeomorphism-induced transformation connected with the identity will suffice. Therefore a finite gauge transformation accomplishing our purpose has the form [10]

$$
V_{\xi}(s, t)=\exp \left(s\left\{-, G_{\xi}(t)\right\}\right),
$$

with a given set of finite descriptors $\xi^{\mu}$. The parameter $s$ labels a trajectory within the gauge orbit. By convention we will assume that at $s=1$ we reach the point $p_{G}$ in the orbit where the gauge-fixing constraints are satisfied. In particular, if we consider the scalars $X^{\mu}(x)$, we will have

$$
\begin{aligned}
X^{\mu}(x) \rightarrow & \hat{X}^{\mu}(x, s)=\exp \left(s\left\{-, G_{\xi}(t)\right\}\right) X^{\mu}(x) \\
= & X^{\mu}(x)+s\left\{X^{\mu}(x), G_{\xi}(t)\right\}+\frac{s^{2}}{2} \\
& \times\left\{\left\{X^{\mu}(x), G_{\xi}(t)\right\}, G_{\xi}(t)\right\}+\ldots,
\end{aligned}
$$

and the gauge-fixing requirement is $\hat{X}^{\mu}(x, 1)=: \hat{X}^{\mu}(x)=$ $x^{\mu}$. (Henceforth "hatted" variables denote variables satisfying the gauge-fixing conditions.) This is an equation that determines the descriptors $\xi(x)$, and we will obtain a unique solution for them in Sec. II B 1. Thus, to any point $p$ in some gauge orbit in $\mathcal{S}$ we associate a system of descriptors. Once the descriptors are determined we can proceed to apply the gauge transformation to all the remaining fields,

$$
\hat{\Phi}^{A}=\exp \left(\left\{-, G_{\xi}\right\}\right) \Phi^{A}=: \mathcal{F}_{\Phi^{A}}[X, \Phi ; \xi] .
$$

\section{Solving for the descriptors}

Let us now solve for the descriptors $\xi^{\mu}$ required in (5). For this purpose it will be convenient to work with the linear combination of secondary first-class constraints that has been introduced by Henneaux and Teitelboim [31] and further exploited by Dittrich [7] and Thiemann [8]. We set, at the fixed coordinate time $t$,

$$
\mathcal{A}^{\mu}{ }_{\nu^{\prime}}:=\left\{X^{\mu}, \mathcal{H}_{\nu^{\prime}}\right\},
$$

with inverse $\mathcal{B}^{\alpha}{ }_{\beta^{\prime}}$, i.e., $\mathcal{B}^{\alpha}{ }_{\beta^{\prime}} \mathcal{A}^{\beta^{\prime}}{ }_{\nu^{\prime \prime}}=\delta_{\nu^{\prime \prime}}^{\alpha}$. [Here we in-

\footnotetext{
${ }^{7}$ If the chosen set of scalars allows for a good coordinatization of the spacetime, no Gribov ambiguities can appear. Let us notice, though, that our considerations are local, and that we can restrict ourselves to a region of the spacetime where the scalar coordinatization works well.
}

troduce the convention that primed indices represent evaluation at primed spatial coordinates, and $\delta_{\nu^{\prime}}^{\mu}:=\delta_{\nu}^{\mu} \delta^{3}\left(x-x^{\prime}\right)$.] We define

$$
\overline{\mathcal{H}}_{\mu}:=\mathcal{B}^{\alpha^{\prime}}{ }_{\mu} \mathcal{H}_{\alpha^{\prime}},
$$

and then rewrite $\xi^{\mu} \mathcal{H}_{\mu}=\bar{\xi}^{\mu} \overline{\mathcal{H}}_{\mu}$, where $\bar{\xi}^{\mu}=$ $\mathcal{A}^{\mu}{ }_{\nu^{\prime}} \xi^{\nu^{\prime}}$. Notice that therefore

$$
\left\{X^{\mu}, \overline{\mathcal{H}}_{\nu^{\prime}}\right\} \approx \mathcal{B}_{\nu^{\prime}}{ }^{\prime \prime} \mathcal{A}^{\mu}{ }_{\alpha^{\prime \prime}}=\delta_{\nu^{\prime}}^{\mu},
$$

where the weak equality signifies that terms proportional to $\mathcal{H}_{\mu}$ have been dropped, or in other words, we evaluate on the original first-class constrained hypersurface. ${ }^{8}$ Of course the change from the original $\mathcal{H}_{\mu}$ to their linear combination $\overline{\mathcal{H}}_{\nu}$ according to (7) is only possible locally.

We will assume that the lapse and shift are not involved in the construction of the scalar fields $X^{\mu}(x)$. In this case only the $\mathcal{H}$ contribution to the generator $G_{\xi}$ in (2) is relevant. Thus the gauge transformed scalar fields, transformed to $p_{G}$, are

$$
\hat{X}^{\mu}(x)=x^{\mu}=\exp \left(\left\{-, \bar{\xi}^{\nu^{\prime}} \overline{\mathcal{H}}_{\nu^{\prime}}\right\}\right) X^{\mu}(x) \approx X^{\mu}(x)+\bar{\xi}^{\mu},
$$

and we can therefore solve on shell for

$$
\bar{\xi}^{\mu}[X(x) ; x]=x^{\mu}-X^{\mu}(x)=: \chi^{\mu}(x),
$$

where $\chi^{\mu}$ are the gauge-fixing constraints introduced in (3).

Although we have obtained a simple closed form for functionals associated with the descriptors in the basis $\overline{\mathcal{H}}_{\nu}$ for the Hamiltonian constraints, the construction of $\overline{\mathcal{H}}_{\nu}$ can of course be difficult in practice due to the need to invert the matrix $\mathcal{A}^{\mu}{ }_{\nu^{\prime}}$. But we will nevertheless be able to prove some interesting formal results in Sec. III.

\section{The observables associated with fields other than the lapse and shift}

We are now in the position to derive an expression for the observables in terms of the gauge-fixing conditions. It is methodologically convenient to first consider the observables associated with fields other than the lapse and shift. This means that the on-shell action of the gauge generator (2) is given just by $\mathcal{H}_{\mu} \xi^{\mu}$. Thus throughout this section, the fields $\Phi^{A}$ do not include the lapse and shift. In Sec. II B 3 this restriction is lifted. We keep working with the basis $\overline{\mathcal{H}}_{\nu}$ for the Hamiltonian constraints, and to make the following considerations easier to follow we temporarily attach a subscript $p$ to the arguments of the

\footnotetext{
${ }^{8}$ An outcome of the construction is that $\left\{\overline{\mathcal{H}}_{\mu}, \overline{\mathcal{H}}_{\nu}\right\}$ is strongly vanishing, instead of weakly vanishing. This result is derived in [8]. We give an alternative symmetry-based proof in Appendix B.
} 
functionals in (5), signifying that they refer to the point $p$ in the gauge orbit,

$$
\hat{\Phi}^{A}=\exp \left(\left\{-, G_{\bar{\xi}_{p}}\right\}\right) \Phi_{p}^{A}=: \mathcal{F}_{\Phi^{A}}\left[X_{p}, \Phi_{p} ; \bar{\xi}_{p}\right],
$$

where $\bar{\xi}_{p}$ are taken as functions of the spacetime coordinates only, whose determination at $p$ is given by $\chi_{p}^{\mu}=$ $x^{\mu}-X_{p}^{\mu}(x)$. Of course, had we started with another point $p^{\prime}$ in the gauge orbit, we would have written

$$
\hat{\Phi}^{A}=\mathcal{F}_{\Phi^{A}}\left[X_{p^{\prime}}, \Phi_{p^{\prime}} ; \bar{\xi}_{p^{\prime}}\right],
$$

with the same functional form because it is the same gauge transformation, see (5), with another set of descriptors. So we have

$$
\mathcal{F}_{\Phi^{A}}\left[X_{p}, \Phi_{p} ; \bar{\xi}_{p}\right]=\mathcal{F}_{\Phi^{A}}\left[X_{p^{\prime}}, \Phi_{p^{\prime}} ; \bar{\xi}_{p^{\prime}}\right] .
$$

Notice that, since they are determined by the field configurations $X_{p}$, the descriptors used to send these field configurations at $p$ to their expressions $\hat{X}=: X_{p_{G}}, \hat{\Phi}=: \Phi_{p_{G}}$, at $p_{G}$ are functionals of $X_{p}$. One can then write, ${ }^{9}$ generally, $\bar{\xi}_{p}^{\mu}(x)=\chi_{p}^{\mu}(x)=x^{\mu}-X_{p}^{\mu}(x)$, and define the new functionals

$$
I_{\Phi^{A}}\left[X_{p}, \Phi_{p} ; x\right]:=\mathcal{F}_{\Phi^{A}}\left[X_{p}, \Phi_{p} ; \bar{\xi}_{p}^{\mu}\right]_{\bar{\xi}_{p}=\chi_{p}} .
$$

It is important to understand that the same functionals $\chi^{\mu}$ work for any point $p$, because $p$ is a generic point in the gauge orbit. That is, for another point $p^{\prime}$, we will have $\bar{\xi}_{p^{\prime}}^{\mu}(x)=\chi_{p^{\prime}}^{\mu}(x)=x^{\mu}-X_{p^{\prime}}^{\mu}(x)$. Thus, using (12),

$$
\begin{aligned}
I_{\Phi^{A}}\left[X_{p}, \Phi_{p} ; x\right] & =\mathcal{F}_{\Phi^{A}}\left[X_{p}, \Phi_{p} ; \bar{\xi}_{p}^{\mu}\right]_{\bar{\xi}_{p}=\chi_{p}} \\
& =\mathcal{F}_{\Phi^{A}}\left[X_{p^{\prime}}, \Phi_{p^{\prime}} ; \bar{\xi}_{p^{\prime}}^{\mu}\right]_{\bar{\xi}_{p^{\prime}}=\chi_{p^{\prime}}} \\
& =\mathcal{I}_{\Phi^{A}}\left[X_{p^{\prime}}, \Phi_{p^{\prime}} ; x\right] .
\end{aligned}
$$

Equation (14) expresses the invariance of the functionals $I_{\Phi^{A}}$. These functionals are observables. In terms of infinitesimal transformations the invariance (14) reads

$$
\left\{I_{\Phi^{A}}, G_{\eta}\right\} \approx 0,
$$

for arbitrary descriptors $\eta$ in $G$. Because of this arbitrariness and the generic form, (2) and (15) is equivalent to

$$
\left\{I_{\Phi^{A}}, \mathcal{H}_{\mu}\right\} \approx 0, \quad\left\{I_{\Phi^{A}}, \mathcal{P}_{\mu}\right\} \approx 0,
$$

and these are the defining conditions for observables. Observe that since $\left\{-, G_{\eta}\right\}$ is tangent to the gauge orbit, the variations of the fields in the functional $I_{\Phi^{A}}$ in (15) are always along the gauge orbit, and thus we need only

\footnotetext{
${ }^{9}$ We assume-and it will prove crucial for the procedure to succeed-that the functionals $\xi^{\mu}$ may carry explicit dependencies on the spacetime coordinates $x^{\mu}$. Remember that when we move from point to point, $p \rightarrow p^{\prime}$ in the gauge orbit through an active diffeomorphism-induced transformation, the spacetime coordinates do not change.
}

information of the functionals on shell to be able to compute (15).

A subtlety not to be overlooked in the definition (13) is the following: the substitution of the descriptors $\bar{\xi}_{p}$ by the gauge-fixing constraints (which do not vanish in $p \neq p_{G}$ ) is made after the functional $\mathcal{F}_{\Phi^{A}}$ has been computed with a descriptor that has no dependence on the fields; or to say it in another way, the descriptors used in (11) have vanishing Poisson brackets with all the fields. More on this will be said in Sec. III C.

At this point, some further comments are in order. First, it is worth noticing that the invariants $I_{\Phi^{A}}$ will in general be nonlocal as regards the spatial coordinates, due to the nesting of commutators in the expansion of the functionals in terms of the fields and their space derivatives; we encounter here a result first obtained by Torre in [6]. Second, citing in advance a result from the following section, there is an invariant associated with any field, including lapse and shift. Third, the method above can also be used to define invariants associated with any functional of the fields. And fourth, the observables $I_{\Phi^{A}}$ can be interpreted in two equivalent ways. On the one hand, an observer with an on-shell field configuration at $p$ has a prescription $\Phi^{A} \rightarrow I_{\Phi^{A}}$ for associating an invariant with any field, and she knows that the description of her solution provided by the invariants will coincide with that of any other observer that uses the same recipe to obtain the invariants, but she always remains at $p$. On the other hand, if such an observer decides to use these functionals in order to work with the new fields $\hat{\Phi}^{A}:=I_{\Phi^{A}}\left[X_{p}, \Phi_{p} ; x\right]$, then this means that she has been able to obtain, with the redefiniton of the fields $\Phi^{A} \rightarrow \hat{\Phi}^{A}$ the description of the observer at $p_{G}$, just reflecting the active view of diffeomorphism transformations. Notice that as long as she decides to work with the new fields $\hat{\Phi}^{A}$-the observables - as the fields of her spacetime, everything in her new description is as if her original coordinates played the role of the intrinsic coordinates. We comment on the complementary passive view in Sec. IIC.

With the explicit solution (10) for the descriptors, the expansion of the invariant $I_{\Phi^{A}}$ in (13) becomes

$$
\begin{aligned}
I_{\Phi^{A}} \approx & \exp \left(\left\{-, \bar{\xi}^{\nu} \overline{\mathcal{H}}_{\nu}\right\}\right) \Phi_{\left.\right|_{\bar{\xi}=\chi} ^{A}} \\
= & \Phi^{A}+\chi^{\mu}\left\{\Phi^{A}, \overline{\mathcal{H}}_{\mu}\right\}+\frac{1}{2 !} \chi^{\mu} \chi^{\nu}\left\{\left\{\Phi^{A}, \overline{\mathcal{H}}_{\mu}\right\}, \overline{\mathcal{H}}_{\nu}\right\} \\
& +\frac{1}{3 !} \chi^{\mu} \chi^{\nu} \chi^{\rho}\left\{\left\{\left\{\Phi^{A}, \overline{\mathcal{H}}_{\mu}\right\}, \overline{\mathcal{H}}_{\nu}\right\}, \overline{\mathcal{H}}_{\rho}\right\}+\ldots \\
= & : \sum_{n=0}^{\infty} \frac{1}{n !} \chi^{n}\{\Phi, \overline{\mathcal{H}}\}_{(n)} .
\end{aligned}
$$

[In the last line we have adopted a simplifying notation where indices in $\chi$ saturate with indices of $\overline{\mathcal{H}}$ and $\{\Phi, \overline{\mathcal{H}}\}_{(n)}$ is interpreted as the repeated nesting of $n$ Poisson brackets with $\overline{\mathcal{H}}$ in the right-hand side]. With 
different notation, this expression appeared in the literature in [8] as his equation (2.8) and in [7] as her equation (5.23). Here we have arrived at (16) by a symmetry-inspired procedure, as the effect of the finite gauge transformation that sends $p$ to $p_{G}$. This specific gauge transformation is determined once the set of scalar fields associated with the gauge fixing has been selected. An advantage of the present formulation is that one can send all the fields from $p$ to $p_{G}$, and this includes the lapse and shift. In this general case one must use the full gauge generator (2) and it is worked out in Sec. II B 3.

The gauge invariance of (16) is guaranteed by the construction procedure, as long as the series expansion is convergent, which is expected at least in a neighborhood of $p_{G}$. Note, that one can directly verify the gauge invariance of $I_{\Phi^{A}}$ by checking the vanishing on shell of $\left\{I_{\Phi^{A}}, \overline{\mathcal{H}}_{\mu}\right\}$ with use of the on-shell expansion (16). It is crucial in this respect, as noticed already in [8], that the Poisson brackets of the constraints $\overline{\mathcal{H}}_{\mu}$ among themselves are quadratic in the constraints (see Appendix B). One can proceed as follows.

Let us define

$$
B_{\Phi}^{(n)}:=\chi^{n}\{\Phi, \overline{\mathcal{H}}\}_{(n)},
$$

with $B_{\Phi}^{(0)}=\Phi$. Then

$$
\left\{B_{\Phi}^{(n)}, \overline{\mathcal{H}}\right\} \approx-n B_{\{\Phi, \overline{\mathcal{H}}\}}^{(n-1)}+B_{\{\Phi, \overline{\mathcal{H}}\}}^{(n)},
$$

from which,

$$
\begin{aligned}
\left\{I_{\Phi}, \overline{\mathcal{H}}_{\mu}\right\} & =\left\{\sum_{n=0}^{\infty} \frac{1}{n !} B_{\Phi}^{(n)}, \overline{\mathcal{H}}_{\mu}\right\} \\
& \approx-\sum_{n=0}^{\infty} \frac{1}{(n-1) !} B_{\{\Phi, \overline{\mathcal{H}}\}}^{(n-1)}+\sum_{n=0}^{\infty} \frac{1}{n !} \\
& =B_{\{\Phi, \overline{\mathcal{H}}\}}^{(n)} \times 0 .
\end{aligned}
$$

It is worth noticing that the proof of invariance given above does not depend of the fact that the gauge-fixing constraints $\chi^{\mu}$ are made up with scalar fields $X^{\mu}$. If, instead of using the scalar fields $X^{\mu}$ in the process to define the basis $\mathcal{H}_{\mu}$ for the Hamiltonian constraints, one uses another set of fields - or field components-the proof of invariance remains intact.

On the other hand, if nonscalars were used for the gauge fixing, it is very likely that Gribov ambiguities will appear. Suppose for instance that the gauge fixing was implemented with a vector $J^{\mu}(x)$, so one should make a change of coordinates $x \rightarrow \hat{x}$ such that $\hat{x}^{\mu}-\hat{J}^{\mu}(\hat{x})=0$. Considering the rules to transform a vector under diffeomorphisms: $J^{\mu}(x) \rightarrow \hat{J}^{\nu}(\hat{x})=J^{\mu}(x) \frac{\partial \hat{x}^{\nu}}{\partial x^{\mu}}$, one should look for a transformation realizing $\hat{J}^{\nu}(\hat{x})=\hat{x}^{\nu}$.

Since the intrinsic vector field is $\vec{J}=J^{\mu}(x) \frac{\partial}{\partial x^{\mu}}$, the equation to obtain the intrinsic coordinates is nothing but
$\vec{J} \hat{x}^{\nu}(x)=\hat{x}^{\nu}(x)$. Thus we look for four eigenfunctions of $\vec{J}$ with unit eigenvalue.

If we have four such eigenfunctions $f^{\mu}(x), \vec{J} f^{\nu}=f^{\nu}$, any linear numerical matrix $A$ will introduce an ambiguity $f^{\mu} \rightarrow \tilde{f}^{\mu}=A_{\nu}^{\mu} f^{\nu}$.

As argued at the beginning of Sec. II A, a proper gauge fixing needs to be performed with spacetime scalars, and we will maintain this requirement throughout.

\section{Observables associated with fields including lapse and shift}

Here we extend the results of the previous section to include the observables associated with the lapse and shift fields.

Recall that the gauge generator (2) is

$$
G_{\xi}(t)=P_{\mu} \dot{\xi}^{\mu}+\left(\mathcal{H}_{\mu}+N^{\rho} C_{\mu \rho}^{\nu} P_{\nu}\right) \xi^{\mu},
$$

where the descriptors $\xi^{\mu}$ are arbitrary functions which may depend, of course, on the coordinates but also on the fields other than lapse and shift. $\dot{\xi}^{\mu}$ is read as

$$
\dot{\xi}^{\mu}=\frac{d}{d t} \xi^{\mu}=\frac{\partial}{\partial t} \xi^{\mu}+N^{\rho}\left\{\xi^{\mu}, \mathcal{H}_{\rho}\right\},
$$

so that the explicit dependence on the time parameter is accounted for in the first term whereas in the second term the implicit time dependence through the fields is reflected through their own dynamics.

When all fields are considered, one must observe that the gauge-fixing constraints $\chi^{\mu}=x^{\mu}-X^{\mu}=: \chi^{(1) \mu}$ have secondary descendants:

$$
\frac{d}{d t} \chi^{\mu}=\delta_{0}^{\mu}-N^{\rho}\left\{X^{\mu}, \mathcal{H}_{\rho}\right\},
$$

and thus the lapse and shift become determined by the secondary gauge-fixing constraints

$$
\chi^{(2) \mu}:=\delta_{0}^{\mu}-\mathcal{A}^{\mu}{ }_{\rho} N^{\rho} \approx 0 .
$$

Preservation of these constraints in time leads to the determination of the arbitrary functions in the Dirac Hamiltonian and the gauge is completely fixed. Note that, by definition, it is only at $p_{G}$ that these gauge-fixing constraints are satisfied.

Now we follow the same steps taken in II B 1, but with the number of constraints doubled. Our 8 gauge-fixing constraints $\chi^{(i) \mu}=\left(\chi^{\mu}, \dot{\chi}^{\mu}\right)$ can be used to change the basis of the 8 first-class constraints, $\zeta_{(j) \nu}=\left(\mathcal{H}_{\nu}, P_{\nu}\right)$ to another basis $\bar{\zeta}_{(j) \nu}=\left(\overline{\overline{\mathcal{H}}}_{\nu}, \bar{P}_{\nu}\right)$ so that $\left\{\chi^{(i) \mu}, \bar{\zeta}_{(j) \nu}\right\} \approx$ $-\delta_{j}^{i} \delta_{\nu}^{\mu}$. These new $\bar{\zeta}_{(i) \mu}$ will have strongly (instead of weakly) vanishing Poisson brackets among themselves at any point $p$ in the gauge orbit. This setting is convenient because it makes possible an easy determination of the descriptors associated with the specific gauge transformation that sends the field configurations at $p$ to their corresponding fields at $p_{G}$. 
The matrix of the gauge-fixing constraints with the firstclass constraints,

$$
\left\{\chi^{(i) \mu}, \zeta_{(j) \nu}\right\}=\left(\begin{array}{cc}
-A^{\mu}{ }_{\nu} & 0 \\
-\left\{\mathcal{A}^{\mu}{ }_{\lambda}, \mathcal{H}_{\nu}\right\} N^{\lambda} & -\mathcal{A}^{\mu}{ }_{\nu}
\end{array}\right),
$$

has the inverse

$$
\begin{aligned}
\mathcal{M}^{i \mu}{ }_{j \nu} & :=\left(\left\{\chi^{(i) \mu}, \zeta_{j \nu}\right\}\right)^{-1} \\
& =\left(\begin{array}{cc}
-B^{\mu}{ }_{\nu} & 0 \\
B^{\mu}{ }_{\lambda} B^{\rho}{ }_{\nu} N^{\sigma}\left\{A^{\lambda}{ }_{\sigma}, \mathcal{H}_{\rho}\right\} & -\mathcal{B}^{\nu}{ }_{\nu}
\end{array}\right),
\end{aligned}
$$

(where $\mathcal{B}^{\mu}{ }_{\rho}$ was defined before, see II B 1, as the inverse matrix of $\mathcal{A}^{\mu}{ }_{\rho}:=\left\{X^{\mu}, \mathcal{H}_{\rho}\right\}$ ) and defines the new basis of first-class constraints as $\bar{\zeta}_{(j) \nu}=-\mathcal{M}^{i \mu}{ }_{j \nu} \zeta_{i \mu}$. We obtain

$$
\bar{P}_{\mu}=\mathcal{B}_{\mu}^{\rho} P_{\rho},
$$

and

$$
\overline{\mathcal{H}}_{\nu}=\mathcal{B}^{\rho}{ }_{\nu}\left(\mathcal{H}_{\rho}-\mathcal{B}^{\mu}{ }_{\lambda} N^{\sigma}\left\{A_{\sigma}^{\lambda}, \mathcal{H}_{\rho}\right\} P_{\mu}\right) .
$$

As a consequence, to express the gauge generator in the new basis we need to implement

$$
\begin{aligned}
& P_{\mu}=\mathcal{A}^{\rho}{ }_{\mu} \bar{P}_{\rho}, \\
& \mathcal{H}_{\rho}=\mathcal{A}^{\nu}{ }_{\rho} \overline{\overline{\mathcal{H}}}_{\nu}+\mathcal{B}^{\mu}{ }_{\lambda} N^{\sigma}\left\{A^{\lambda}{ }_{\sigma}, \mathcal{H}_{\rho}\right\} P_{\mu} .
\end{aligned}
$$

With these substitutions, the gauge generator (2) becomes

$$
\begin{aligned}
G_{\xi}(t)= & \mathcal{A}^{\nu}{ }_{\mu} \bar{P}_{\nu} \dot{\xi}^{\mu}+\left(\mathcal{A}^{\nu}{ }_{\mu} \overline{\overline{\mathcal{H}}}_{\nu}\right. \\
& \left.+\mathcal{B}^{\lambda}{ }_{\rho} N^{\sigma}\left\{A^{\rho}{ }_{\sigma}, \mathcal{\mathcal { H }}{ }_{\mu}\right\} P_{\lambda}+N^{\sigma} C_{\mu \sigma}^{\lambda} P_{\lambda}\right) \xi^{\mu} .
\end{aligned}
$$

Now we may consider the special case when $\xi^{\sigma}$ is such that $\bar{\xi}^{\nu}:=\mathcal{A}^{\nu}{ }_{\sigma} \xi^{\sigma}$ is field independent. We have

$$
\dot{\xi}^{\mu}=\frac{d}{d t}\left(\mathcal{B}^{\mu}{ }_{\sigma} \bar{\xi}^{\sigma}\right)=\left\{\mathcal{B}^{\mu}{ }_{\sigma}, N^{\lambda} \mathcal{H}_{\lambda}\right\} \bar{\xi}^{\sigma}+\mathcal{B}^{\mu}{ }_{\sigma} \dot{\bar{\xi}}^{\sigma},
$$

and the gauge generator becomes

$$
G_{\bar{\xi}}(t)=\bar{P}_{\nu} \dot{\bar{\xi}}^{\nu}+\overline{\overline{\mathcal{H}}}_{\nu} \bar{\xi}^{\nu}+P_{\mu} N^{\sigma} \mathcal{S}_{\rho \sigma}^{\mu} \bar{\xi}^{\rho},
$$

where $\mathcal{S}_{\rho \sigma}^{\mu}$ is defined as

$$
\mathcal{S}_{\rho \sigma}^{\mu}=\left\{\mathcal{B}^{\mu}{ }_{\rho}, \mathcal{H}_{\sigma}\right\}+\mathcal{B}^{\nu}{ }_{\rho} \mathcal{B}^{\mu}{ }_{\gamma}\left\{\mathcal{A}^{\gamma}{ }_{\sigma}, \mathcal{H}_{\nu}\right\}+\mathcal{B}^{\nu}{ }_{\rho} C_{\nu \sigma}^{\mu} .
$$

But $\mathcal{S}_{\rho \sigma}^{\mu}$ is just a constraint, in fact a linear combination of the Hamiltonian constraints. To see this let us use the fact that the matrices $\mathcal{A}, \mathcal{B}$, (with discrete and continuous indices as well) are inverses to each other. We obtain

$$
\begin{aligned}
\mathcal{A}^{\beta}{ }_{\mu} \mathcal{A}{ }_{\alpha} \mathcal{S}_{\rho \sigma}^{\mu}= & -\left\{\mathcal{A}^{\beta}{ }_{\alpha}, \mathcal{H}_{\sigma}\right\}+\left\{\mathcal{A}^{\beta}{ }_{\sigma}, \mathcal{H}_{\alpha}\right\} \\
& +C_{\alpha \sigma}^{\mu} \mathcal{A}^{\beta}{ }_{\mu},
\end{aligned}
$$

which, using the definition (6) of $\mathcal{A}^{\beta}{ }_{\mu}$, becomes

$$
\begin{aligned}
\mathcal{A}^{\beta}{ }_{\mu} \mathcal{A}^{\rho}{ }_{\alpha} \mathcal{S}_{\rho \sigma}^{\mu} & =-\left\{X^{\beta},\left\{\mathcal{H}_{\alpha}, \mathcal{H}_{\sigma}\right\}\right\}+C_{\alpha \sigma}^{\mu}\left\{X^{\beta}, \mathcal{H}_{\mu}\right\} \\
& =-\left\{X^{\beta}, C_{\alpha \sigma}^{\mu}\right\} \mathcal{H}_{\mu},
\end{aligned}
$$

which proves our assertion. The gauge generator in the new basis is therefore $G_{\bar{\xi}}(t)=\bar{P}_{\nu} \dot{\bar{\xi}}^{\nu}+\overline{\mathcal{H}}_{\nu} \bar{\xi}^{\nu}+\mathcal{O}(2)$, where by $\mathcal{O}(2)$ we mean terms that are quadratic in the constraints. Since we always work on shell, this last term is irrelevant and we discard it, obtaining a very simple expression for the gauge generator in the new basis,

$$
G_{\bar{\xi}}(t)=\bar{P}_{\nu} \dot{\bar{\xi}}^{\nu}+\overline{\mathcal{H}}_{\nu} \bar{\xi}^{\nu} .
$$

Finally, imposing the usual condition: $\exp \left(\left\{-, G_{\bar{\xi}}\right\}\right) X^{\mu}=\hat{X}^{\mu}=x^{\mu}$, we can determine the descriptors that must be employed to transform from $p$ to $p_{G}$. Their functional form on shell is

$$
\bar{\xi}^{\sigma} \rightarrow \chi^{\sigma}=x^{\sigma}-X^{\sigma}, \quad \dot{\bar{\xi}}^{\sigma} \rightarrow \dot{\chi}^{\sigma}=\delta_{0}^{\sigma}-N^{\nu} A^{\sigma}{ }_{\nu} .
$$

Thus for any field, including lapse and shift, the invariants are defined as in Sec. II B 2 but with the full gauge generator $G_{\bar{\xi}}$ given in (24) and with the substitutions for the descriptors implemented after the action of the gauge generator, as prescribed above.

\section{Passive coordinate transformation from $p$ to $p_{G}$}

There must exist passive coordinate transformations that correspond to the active transformation to the gauge-fixed point $p_{G}$ on the gauge orbit, and we can easily find them. Let us suppose that this passive transformation takes the functional form $\hat{x}^{\mu}=f^{\mu}(x)$. Since by assumption $X^{\mu}(x)$ transforms as a spacetime scalar under this transformation, we have $\hat{X}^{\mu}(\hat{x})=X^{\mu}(x)$, so that the transformed fields at the original coordinate location $x^{\mu}$ are $\hat{X}^{\mu}(x)=x^{\mu}=$ $X^{\mu}\left(f^{-1}(x)\right)$. Equivalently

$$
f^{\mu}(x)=X^{\mu}(x) .
$$

In other words, and this is one of our key observations, the performance of the gauge transformation-active viewto the solutions satisfying the gauge conditions is equivalent to the performance of a coordinate transformationpassive view-from the original coordinate system to intrinsic coordinates. Every dynamical field evaluated in this intrinsic coordinate system will therefore be an invariant under diffeomorphism-induced gauge transformations, and this includes the lapse and shift.

Considering, in particular, the case of a scalar field $\Psi$, as described by the user sitting at $p$, the prescription dictated by the passive coordinate transformation is $\hat{\Psi}(x)=$ $\Psi\left(X^{-1}(x)\right)$ or, equivalently, $\Psi_{p_{G}}(x)=\Psi_{p}\left(X^{-1}(x)\right)$. In the case of other, nonscalar, fields, they transform according to their geometric properties. These observables may be displayed as power series in the coordinates $x^{\mu}$ in the following manner. Repeated derivatives of the identities $X^{-1 \mu}(X(x))=x^{\mu}$ followed by substitution of the 
Hamiltonian equations of motion will yield a Taylor ex-

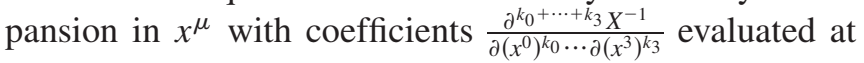
$x^{\mu}=X^{\mu}$. The resulting expansions can be obtained in a more efficient manner through active transformations, as we now show.

\section{PROPERTIES OF THE OBSERVABLES}

\section{A. Dynamics of the observables}

\section{The equations of motion for the invariants $I_{\Phi}$}

The explicit dependence on time- through the determination of the descriptors (10) — makes the observables (16) time dependent; the implicit dependence is canceled due to the invariance $\left\{I_{\Phi}, \mathcal{H}\right\} \approx 0$. Considering fields $\Phi$ other than lapse and shift (for which the following considerations can be extended appropriately),

$$
\begin{aligned}
\frac{d}{d t} I_{\Phi}= & \frac{\partial}{\partial t} I_{\Phi}+\left\{I_{\Phi}, N^{\mu} \mathcal{H}_{\mu}\right\} \approx \frac{\partial}{\partial t} I_{\Phi} \\
\approx & \left\{\Phi, \overline{\mathcal{H}}_{0}\right\}+\frac{1}{2}\left(\chi^{0} \chi^{\nu}\left\{\left\{\Phi, \overline{\mathcal{H}}_{0}\right\}, \overline{\mathcal{H}}_{\nu}\right\}\right. \\
& \left.+\chi^{\mu} \chi^{0}\left\{\left\{\Phi, \overline{\mathcal{H}}_{\mu}\right\}, \overline{\mathcal{H}}_{0}\right\}\right)+\cdots \\
\approx & \sum_{n=0}^{\infty} \frac{1}{n !} \chi^{n}\left\{\left\{\Phi, \overline{\mathcal{H}}_{0}\right\}, \overline{\mathcal{H}}\right\}_{(n)}=I_{\left\{\Phi, \overline{\mathcal{H}}_{0}\right\}}
\end{aligned}
$$

where in the second line we have used the strong vanishing of the Poisson brackets of the $\overline{\mathcal{H}}$, cf. Appendix B. Thus the equations of motion for the invariants are

$$
\frac{d}{d t} I_{\Phi} \approx I_{\left\{\Phi, \overline{\mathcal{H}}_{0}\right\}}
$$

The appearance of $\overline{\mathcal{H}}_{0}$ in (27) might come as a surprise, but it is exactly what is needed in order for the fields at $p_{G}$ to satisfy the equations of motion. In fact, starting at any point $p$ in $\mathcal{S}$, the invariants produce the corresponding fields at $p_{G}$ : $I_{\Phi}\left[X_{p}, \Phi_{p} ; x\right]=\Phi_{p_{G}}, \quad$ and $\mathcal{I}_{\left\{\Phi, \overline{\mathcal{H}}_{0}\right\}}\left[X_{p}, \Phi_{p} ; x\right]=\left\{\Phi, \overline{\mathcal{H}}_{0}\right\}_{p_{G}}$. On the other hand, from (7) we know that $\overline{\mathcal{H}}_{0}=\mathcal{B}^{\mu}{ }_{0} \mathcal{H}_{\mu}$. Also, at $p_{G}$, the dynamical stabilization of the gauge-fixing constraints introduces the new constraints (19), which may be written as

$$
N^{\mu}-\mathcal{B}^{\mu}{ }_{0} \approx 0,
$$

thus fixing the values of lapse and shift in terms of other fields. Note then that, when computed at $p_{G}, \overline{\mathcal{H}}_{0} \equiv$ $N^{\mu} \mathcal{H}_{\mu}$, where we use Dirac's strong equality symbol, defined in Appendix B. Thus, the content of (27) is just

$$
\frac{d}{d t} \Phi_{p_{G}} \approx\left\{\Phi, N^{\mu} \mathcal{H}_{\mu}\right\}_{p_{G}},
$$

which is nothing but the equations of motion for the fields - other than lapse and shift—at $p_{G}$. This result can be extended for the lapse and shift fields by using techniques described in Sec. II B 3.
This fact that observables are time dependent, already stressed in [10], contradicts claims of standard lore asserting that observables are compelled to be constants of motion because they have vanishing Poisson brackets with the generator of time evolution. The simple mistake in this claim is that it does not take into account the compulsory explicit time dependence ${ }^{10}$ that is needed for a correct gauge fixing in generally covariant theories, which has been proven in $[27,28]$. Deeply connected with this mistake is the confusion between gauge symmetry and dynamical evolution, also common in the literature and to which we devote the Appendix A.

\section{From observables to "evolving constants of motion"}

We continue to work for simplicity with fields other than the lapse and shift. We shall show now how constants of motion-and Noether generators-without explicit time dependence can be easily recovered form our program.

Let us stress again the fact that the time dependence in the invariants $I_{\Phi},(16)$, has two sources. One is the explicit time dependence in $\chi^{0}=t-X^{0}$, and the other is the implicit time dependence in the on-shell field configuration (including the scalars $X^{\mu}$ ). But we have already seen at the beginning of the previous section that this implicit time dependence is nonexistent because of the gauge invariance. In fact, it is easy to separate these two dependencies and witness the difference. For instance one could write $I_{\Phi}$ with an "explicit" time $t_{1}$ and an "implicit"-i.e., the time argument in the field configuration-time $t_{2}$ and eventually realize ${ }^{11}$ that the only true dependence is in $t_{1}$. This result suggests that we can consider the invariants as a oneparameter family of functionals in phase space, which can be evaluated on on-shell field configurations at an arbitrary time $t_{2}$. Notice that as long as we keep $t_{1}$ fixed, the invariants do indeed yield constants of motion.

Up to now our arena regarding the distinction between explicit and implicit time dependence has been the space $\mathcal{S}$ of on-shell field configurations. Now, continuing with our previous construction we move to phase space, where the variables are the fields exhibiting only spatial dependence on the coordinates. Let us examine the invariants $I_{\Phi}$ in phase-space and its remaining dependence on the explicit time. In fact, using (27) and differentiating repeatedly with respect to the explicit time, we obtain

$$
\frac{\partial^{n}}{\partial t^{n}} I_{\Phi} \approx I_{\left\{\Phi, \overline{\mathcal{H}}_{0\}(n)}\right.} .
$$

where $\left\{\Phi, \overline{\mathcal{H}}_{0}\right\}_{(n)}$ has the usual sense of nested Poisson

\footnotetext{
${ }^{10}$ One can recall here the case of Galilean mechanics, were the boost generators are constants of motion with explicit time dependence, and therefore their Poisson bracket with the Hamiltonian is nonvanishing.

${ }^{11}$ Note that for each point $p$ in $\mathcal{S}$, and given $t_{1}, t_{2}$, there is a point $p^{\prime}$ where $\Phi_{p}\left(t_{2}, \vec{x}\right)=\Phi_{p^{\prime}}\left(t_{1}, \vec{x}\right)$, for any field or field component $\Phi$.
} 
brackets. From (30) we can build the Taylor expansion in the $t$ parameter

$$
I_{\Phi} \approx \sum_{n=0}^{\infty} \frac{t^{n}}{n !} I_{\left\{\Phi,\left.\overline{\mathcal{H}}_{0\}(n)}\right|_{t=0} .\right.} .
$$

(Recall that we think of these quantities in phase space, i.e., the invariants are now dependent on the phase-space variables, these phase-space variables being fields depending on the spatial coordinates.) Notice that the coefficients of this expansion in the time parameter are constants of motion, because they are invariants; since they have vanishing Poisson brackets with the Hamiltonians they have no explicit time dependence. In fact, as pointed out before, fixing the explicit time parameter at any arbitrary value, the invariants $I_{\Phi}$ become constants of motion.

One may wonder what is then the role of this explicit time dependence in the invariants? The answer has been given in Eq. (29), which is a consequence of (27). It is remarkable that the explicit time dependence in (31) allows us to fully recover the dynamics for the configuration of the fields that satisfy the gauge fixing. Thus, two observers using the same recipe to construct the invariants will be able to describe with the help of these invariants the same physics at any time - which is their own original time coordinate-because the dynamical evolution is already built into the invariants thanks to the explicit time dependence. With their invariants, they will describe physical evolution in an invariant way, through the explicit time dependence. Notice that from the point of view of a typical observer, sitting at some point $p$ in $\mathcal{S}$, the explicit time parameter appearing in his/her observables $I_{\Phi}$ is just his/ her original time parameter, that is, a labeling for the spacelike hypersurfaces foliating the spacetime-or at least a region of it —satisfying the rather mild requirement of being an increasing function from past to future. In addition, for the observer sitting at $p_{G}$, this explicit time parameter is the value of the scalar field $X^{0}$, that is, the intrinsic time.

One may regard expression (31) for the invariants as an expression for "evolving constants of motion" since it can be read as a combination of constants of motion with coefficients-the monomials $t^{n}$-changing in time. As a matter of fact, expression (31) is an explicit realization of the approach [15-17], which is often referred to as the program of "evolving constants of motion." We believe that the clarifications made above, although restricted to classical considerations, put in a new and satisfying perspective the concept of "evolving constants of motion." This terminology was adopted in order to circumvent a problem which, from our perspective, never existed in the first place-at least in the classical setting. The problemthe notion that "nothing happens" in generally covariant theories-arises from a failure to adequately distinguish between time evolution and gauge symmetry. We devote Appendix A to this analysis. We think that our contribution makes superfluous this terminology, although the idea behind it remains fully vindicated. The resolution by Rovelli of the supposedly apparent paradox amounts to [32] "the recognition that observables are members of families of constants of motion parametrized by a label related to time." In other words, as the time coordinate for a particular observer evolves and takes different values, so do the observables. The observables consist of a sumperhaps even of an infinite number of terms - of constants of the motion multiplying increasing powers of the time coordinate. These constant coefficients are expressed as invariant functionals of the phase-space variables. The coordinate time itself is of course not a canonical variable, and is therefore invariant under the action of the canonical gauge group. There has been a subsequent effort by a list of authors, particularly [8,33], to obtain a consistent quantum picture for the observables. In generic spacetimes it will be necessary to employ a locally defined intrinsic time. One must also address the question of equivalence of quantum theories based on different choices.

\section{From observables to generators of rigid Noether symmetries}

Consider the observables associated with fields other than lapse and shift. The constants of motion obtained from these observables are Noether generators of symmetries for the reduced phase space where lapse, shift, and their canonical momenta have been eliminated. If we want to construct Noether generators for the entire phase space, one can proceed as follows. Let $\mathcal{C}$ be one of such constants of motion. It is a functional of the fields $\Phi(\vec{x})$-other than lapse and shift-and it exhibits explicit dependence on the spatial coordinates $\vec{x}$ as well, through $\chi^{i}=x^{i}-X^{i}$. Its weakly vanishing Poisson bracket with the Hamiltonian constraints can be expressed as

$$
\left\{\mathcal{C}, \mathcal{H}_{\mu}\right\}=U^{\nu}{ }_{\mu} \mathcal{H}_{\nu},
$$

for some functional matrix $U^{\nu}{ }_{\mu}$. Then the following construction

$$
\mathcal{Q}:=\mathcal{C}+U_{\mu}^{\nu} N^{\mu} P_{\nu},
$$

satisfies $\left(H_{c}\right.$ stands for Dirac's canonical Hamiltonian, $H_{c}=N^{\mu} \mathcal{H}_{\mu}$ )

$$
\frac{\partial \mathcal{Q}}{\partial t}+\left\{\mathcal{Q}, H_{c}\right\}=0+\left\{\mathcal{Q}, N^{\mu} \mathcal{H}_{\mu}\right\}=\mathcal{O}(P),
$$

and

$$
\left\{\mathcal{Q}, P_{\mu}\right\}=\mathcal{O}(P),
$$

[where $\mathcal{O}(P)$ means terms linear in the momentum constraints] which are the two conditions spelled out in [34] for $\mathcal{Q}$ to qualify as a Noether conserved quantity associated with a symmetry which is projectable from tangent 
space to phase space. In fact this symmetry is generated by $\mathcal{Q}$ through the Poisson bracket. ${ }^{12}$

Notice that these Noether generators $\mathcal{Q}$ are gauge invariant quantities because their Possion bracket with the full set of primary and secondary constraints weakly vanishes. In practice, to smooth out the dependence of $Q$ on the spatial coordinates, one can use arbitrary smearing functions $\alpha(\vec{x})$ and define generators

$$
\mathcal{Q}_{\alpha}:=\int d^{3} x \alpha(\vec{x}) Q(\vec{x})
$$

Let us stress that $\mathcal{Q}_{\alpha}$ are generators of rigid Noether symmetries. To be gauge symmetries one should allow the functions $\alpha$ to have arbitrary dependence on the time coordinate, but if we allow that then $\mathcal{Q}_{\alpha}$ ceases to be a constant of motion and a Noether generator.

As regards the constants of motion extracted from the observables associated with the lapse and shift, one should consider $I_{N^{\sigma}}=\exp \left(\left\{-, G_{\bar{\xi}}\right\}\right) N^{\sigma}$, with $G_{\bar{\xi}}$ as in (24). This gives an expansion

$$
\begin{aligned}
I_{N^{\sigma}} & =N^{\sigma}+\left(\left\{N^{\sigma}, \bar{P}_{\nu} \dot{\bar{\xi}}^{\nu}\right\}+\ldots\right)_{\bar{\xi}^{\sigma} \rightarrow \chi^{\sigma}, \dot{\bar{\xi}}^{\sigma} \rightarrow \dot{\chi}^{\sigma}} \\
& =\mathcal{B}_{0}^{\sigma}+\ldots,
\end{aligned}
$$

where we have used $\dot{\chi}^{\sigma}=\delta_{0}^{\sigma}-N^{\nu} \mathcal{A}^{\sigma}{ }_{\nu}$. In fact there is a quick shortcut to sum this expansion, because we know that in $p_{G}$ the-secondary-gauge-fixing constraints are satisfied, i.e., $\delta_{0}^{\sigma}-\hat{N}^{\nu} \hat{\mathcal{A}}^{\sigma}{ }_{\nu}=0$, and therefore $\hat{N}^{\sigma}=I_{N^{\sigma}}=$ $I_{\mathcal{B}_{0}^{\sigma}}$. Since $\mathcal{B}_{0}^{\sigma}$ depends on fields other than lapse and shift, we can conclude that using the mechanism explained above in this subsection, the constants of motion extracted from all our invariants can be made Noether symmetry generators.

Finally, let us discuss one more basic aspect of the rigid symmetries generated by $\mathcal{Q}_{\alpha}$ : they move an on-shell field configuration out of its gauge orbit. In fact, since they commute with the gauge generators, they will map an entire gauge orbit into another. To prove that indeed $\mathcal{Q}_{\alpha}$ moves a field configuration out of the gauge orbit we only need to verify that the variations generated by $\mathcal{Q}_{\alpha}$ through the Poisson bracket do not leave invariant the set of gauge invariant functionals $I_{\Phi}$. This is proven in Sec. III B, where we compute the Poisson-bracket algebra of the invariants and obtain a nontrivial result.

\section{Interpreting the constants of motion}

What has been done for the time coordinate may be done for any other coordinate. Recall that the invariant associated with the field $\phi$ is

\footnotetext{
${ }^{12}$ Note, in particular, that $\mathcal{Q}$ generates variations for the lapse and shift according to $\delta N^{\mu}=\left\{N^{\mu}, Q\right\}=U^{\mu}{ }_{\nu} N^{\nu}$.
}

$$
I_{\phi} \approx \sum_{n=0}^{\infty} \frac{1}{n !} \chi^{n}\{\phi, \mathcal{H}\}_{(n)},
$$

with $\chi=x-X$. When the explicit time coordinate is fixed at an arbitrary value, $I_{\phi}$ becomes a constant of motion.

Applying arguments similar to those leading up to (27) we find that

$$
\frac{d}{d x^{a}} I_{\Phi} \approx I_{\left\{\Phi, \overline{\mathcal{H}}_{a}\right\}}
$$

Recall that the invariant $I_{\Phi}$ is the field $\Phi$ evaluated at the gauge-fixed point $p_{G}, I_{\Phi}=\hat{\Phi}$. To find $\overline{\mathcal{H}}_{a}$ at $p_{G}$ we need to make use of the fact that the $X^{\mu}$ are scalars under spatial coordinate transformations, i.e., under $\bar{x}^{\mu}=x^{\mu}-\xi^{a} \delta_{a}^{\mu}$,

$$
\delta X^{\mu}=\left\{X^{\mu}, \int d^{3} x \xi^{a} \mathcal{H}_{a}\right\}=X_{, a}^{\mu} \xi^{a},
$$

and therefore $\mathcal{A}_{a}^{\mu}=X_{, a}^{\mu}$. As a consequence $\left.\mathcal{A}_{a}^{\mu}\right|_{p_{G}}=\delta_{a}^{\mu}$. Taking into account that according to (28) $\left.\mathcal{B}_{0}^{\mu}\right|_{p_{G}}=N^{\mu}$ we find that

$$
\left.\mathcal{B}_{a}^{\mu}\right|_{p_{G}}=\delta_{a}^{\mu} .
$$

It follows finally that

$$
I_{\left\{\Phi, \overline{\mathcal{H}}_{a}\right\}}=\left.\frac{\partial \Phi}{\partial x^{a}}\right|_{p_{G}} .
$$

We know that due to gauge invariance, there is no implicit dependence on time (when the invariant is evaluated on an on-shell configuration, a solution of the equations of motion). For the same reason, there is no implicit dependence on the spatial coordinates either since spatially constant translations are gauge transformations. Let us write $I_{\Phi}$ in powers of all explicit coordinates,

$$
I_{\phi} \approx \sum_{n_{\mu}=0}^{\infty} \frac{1}{n_{0} ! n_{1} ! n_{2} ! n_{3} !}\left(x^{0}\right)^{n_{0}}\left(x^{1}\right)^{n_{1}}\left(x^{2}\right)^{n_{2}}\left(x^{3}\right)^{n_{3}} \mathcal{C}_{n_{0}, n_{1}, n_{2}, n_{3}},
$$

with

$$
\mathcal{C}_{n_{0}, n_{1}, n_{2}, n_{3}}:=I_{\left\{\left\{\left\{\left\{\phi,, \overline{\mathcal{H}}_{0}\right\}_{\left(n_{0}\right)}, \overline{\mathcal{H}}_{1\}}\right\}_{\left(n_{1}\right)}, \overline{\mathcal{H}}_{2\}}\right\}_{\left(n_{2}\right)}, \overline{\mathcal{H}}_{3}\right\}\left(n,\left.\left.3_{3}\right|_{x}\right|^{\mu}=0\right.} .
$$

(The order of the Hamiltonians is irrelevant owing to the strongly vanishing Poisson-bracket property.) Notice that setting $x^{\mu}=0$ in (35) refers only to the explicit coordinate dependencies. Indeed, the coefficients $\mathcal{C}_{n_{0}, n_{1}, n_{2}, n_{3}}$ may be evaluated in any arbitrary system of coordinates; they are invariants. Consider, for example, that we are in a point $p$ in $\mathcal{S}$. To evaluate the value of the functional $\mathcal{C}_{n_{0} n_{1} n_{2} n_{3}}$ we just substitute in the field configurations $\phi_{p}^{A}(x)$ and an indefinite number of their spatial derivatives-which appear due to the nesting of Poisson brackets-in (35). The result does not depend on the time coordinate $x^{0}$ nor on the spatial coordinates $\vec{x}$ where all the fields and their spatial derivatives are computed. If instead of inserting $\phi_{p}^{A}(x)$, 
$\partial_{i} \phi_{p}^{A}(x), \partial_{i} \partial_{j} \phi_{p}^{A}(x) \ldots \forall A$, we were to substitute in the onshell field configuration at another point $q$ in $\mathcal{S}$ and with other values $y$ of the coordinates, $\phi_{q}^{A}(y), \partial_{i} \phi_{q}^{A}(y)$, the numerical result would be the same.

Generalizing (33), we deduce that these constants of motion $\mathcal{C}_{n_{0}, n_{1}, n_{2}, n_{3}}$ are the values of the corresponding $n_{\mu}$ partial derivatives of $\hat{\phi}$ at the zero value of the intrinsic coordinates. (In fact one could have expanded around any other values.) Thus the formalism manages to pick data at any time, $t$, at some point $p$ in $\mathcal{S}$, and to convert these data into the coefficients of the Taylor expansion for the fields at $p_{G}$, which are obviously invariants. By the same token, the information in $I_{\phi}$ itself is that of the field configuration at $p_{G}$ at the given time that appears in the invariant as the explicit time. In the particular case of a scalar field and for an observer sitting at $p, I_{\psi}(t, \vec{x})$ it is the value of the field at the very moment and place where the gauge-fixed scalars $X^{\mu}$ take the values $t, \vec{x}$.

\section{A simple example}

Now we demonstrate with the simplest of the examples, that of the massive free particle in Minkowski spacetime, our findings above. ${ }^{13}$ Consider the Lagrangian

$$
L=\frac{1}{2 N} \eta_{\mu \nu} \dot{q}^{\mu} \dot{q}^{\nu}-\frac{1}{2} m^{2} N
$$

where $N$ is an auxiliary variable - in fact it is the lapseand $\eta^{\mu \nu}=(-,+,+,+)$. The Dirac Hamiltonian is

$$
H_{D}=\frac{1}{2} N\left(\eta^{\mu \nu} p_{\mu} p_{\nu}+m^{2}\right)+\lambda \pi
$$

where $\pi$, the momentum canonically conjugate to $N$, is the primary constraint and $\lambda$ an arbitrary function of time. There is a secondary constraint, namely, $\mathcal{H}=\frac{1}{2} \times$ $\left(\eta^{\mu \nu} p_{\mu} p_{\nu}+m^{2}\right)$. The gauge generator has the form $G=$ $\xi \mathcal{H}+\dot{\xi} \pi$. We choose as a gauge-fixing constraint $\chi=$ $t-q^{0}$. Next, following the instructions in Sec. II B 1, we define $A:=\left\{q^{0}, \mathcal{H}\right\}=p^{0}$, and

$$
\overline{\mathcal{H}}=\frac{1}{A} \mathcal{H}=\frac{1}{2 p^{0}}\left(\eta^{\mu \nu} p_{\mu} p_{\nu}+m^{2}\right)
$$

Now we are ready to compute the invariants. Note that we do not write the implicit time dependence in the variables, which is the same as if we were working just in phase space instead of working in the space of trajectories, i.e., field configurations. The series expansions are trivial and we get

\footnotetext{
${ }^{13}$ The example of a dimensionally reduced spatially homogeneous isotropic cosmological model is worked out in [12].
}

$$
\begin{aligned}
I_{q^{i}} & =q^{i}+\chi\left\{q^{i}, \overline{\mathcal{H}}\right\}=q^{i}+\left(t-q^{0}\right) \frac{p^{i}}{p^{0}} \\
& =\left(q^{i}-\frac{p^{i}}{p^{0}} q^{0}\right)+\frac{p^{i}}{p^{0}} t, \\
I_{p_{\mu}} & =p_{\mu} .
\end{aligned}
$$

Thus we identify from the expansion in the $t$ parameter for $I_{q^{i}}$ the constants of motion $q^{i}-\frac{p^{i}}{p^{0}} q^{0}$ and $\frac{p^{i}}{p^{0}}$, and from $I_{p_{\mu}}$ the constants of motion $p_{\mu}$. We have seven independent constants of motion that can be written $p_{\mu}$ and $c^{i}:=$ $p^{0} q^{i}-q^{0} p^{i}$. These are the Poincarè translation and boost generators. Note that the combinations $\frac{1}{p^{0}}\left(p^{i} c^{j}-p^{j} c^{i}\right)$ of these constants of motion are $p^{i} q^{j}-p^{j} q^{i}$, that is, the generators of rotations. The full Poincaré algebra of generators of rigid symmetries of the free particle is obtained.

Finally, using the methods introduced in Sec. II B 3, one can compute the invariant associated with the lapse $N$. The result is $I_{N}=\frac{1}{p^{0}}$, that is, one of the constants of motion obtained above.

\section{B. Observables and dirac brackets}

\section{Preliminary remarks}

It is a remarkable fact that for some purposes the explicit construction of invariants for which the general theory has been given above can be avoided. In this subsection we will show that the Poisson bracket $\left\{I_{\Phi^{A}}, I_{\Phi^{B}}\right\}$ of the invariants associated with the fields $\Phi^{A}, \Phi^{B}$, is the invariant associated with the Dirac bracket of the fields themselves.

As a preliminary observation, one might wonder how we can compute Poisson brackets of the functional invariants $I_{\Phi^{A}}$, given that their arguments are only defined for fields satisfying the equations of motion; Poisson brackets involve arbitrary variations, including "off shell", i.e., violating the equations of motion. The resolution is the following. Since the Poisson brackets are an equal-time computation, let us simply examine the functionals at a given time $t$. An arbitrary extension off shell of a functional $I_{\Phi^{A}}$ will produce $I_{\Phi^{A}} \rightarrow I_{\Phi^{A}}+\mathcal{O}\left(P_{\mu}, \mathcal{H}_{\nu}\right)$. But notice that this off shell extension does not change the Poisson brackets as long as we evaluate the result on shell since $\left\{\mathcal{O}\left(P_{\mu}, \mathcal{H}_{\nu}\right), \mathcal{F}_{\Phi}\right\} \approx 0$ and also of course $\left\{\mathcal{O}\left(P_{\mu}, \mathcal{H}_{\nu}\right), \mathcal{O}\left(P_{\rho}, \mathcal{H}_{\sigma}\right)\right\} \approx 0$. So indeed the functionals need only be defined on shell for their Poisson bracket to be well defined on shell.

We will show that

$$
\left\{I_{\Phi^{A}}, I_{\Phi^{B}}\right\} \approx I_{\left\{\Phi^{A}, \Phi^{B}\right\}^{*}},
$$

where the Dirac bracket is

$$
\left\{\Phi^{A}, \Phi^{B}\right\}^{*}:=\left\{\Phi^{A}, \Phi^{B}\right\}-\left\{\Phi^{A}, C^{i}\right\} M_{i j}^{-1}\left\{C^{j}, \Phi^{B}\right\} .
$$

In this expression we define the eight member set $C^{i}:=$ $\mathcal{H}_{\mu}, \chi^{\nu}$, and $M^{i j}:=\left\{C^{i}, C^{j}\right\}$. 
Notice that if the map $\Phi^{A} \rightarrow I_{\Phi^{A}}$ were a canonical transformation, the result would have been simply $\left\{I_{\Phi^{A}}, I_{\Phi^{B}}\right\}=I_{\left\{\Phi^{A}, \Phi^{B}\right\}}$, because the Poisson-bracket structure is preserved by a canonical transformation. The crucial fact that complicates this computation is that the descriptors, which are determined by the gauge-fixing conditions, are substituted by functionals of the field configurations at $p$ after the action of the finite element of the gauge group $\exp (\{-, G\})$ is taken, as it is clear in (16). We further discuss in IIIC this issue of noncanonicity of the map $\Phi^{A} \rightarrow I_{\Phi^{A}}$

The following proof is restricted, just for simplicity, to canonical fields other than the lapse and shift and their conjugates. Thus for the generator (2) we only need the reduced expression $G=\bar{\xi}^{\mu} \overline{\mathcal{H}}_{\mu}$, with $\overline{\mathcal{H}}_{\mu}$ defined in (7). This restriction is easily eliminated by taking into account the results in Sec. II B 3 and using the generator (24). The proof will be undertaken in two steps. In the first step we show that this relation holds at $p_{G}$, and in the second step the proof is extended to an arbitrary point in the gauge orbit.

\section{Step 1: Neighborhood of $p_{G}$}

Let us consider a neighborhood of $p_{G}$ in the gauge orbit, and take an arbitrary point $p$ in the same orbit, such that the set of descriptors used to bring configurations in $p$ to configurations in $p_{G}$ are infinitesimal. Let us write, recalling (16) and keeping terms to first order in the infinitesimal descriptors,

$$
I_{\Phi^{A}}=\Phi^{A}+\chi^{\mu}\left\{\Phi^{A}, \overline{\mathcal{H}}_{\mu}\right\}+\mathcal{O}(\chi)^{2} .
$$

Then, computing at $p$,

$$
\begin{aligned}
\left\{I_{\Phi^{A}}, I_{\Phi^{A}}\right\}= & \left\{\Phi^{A}+\chi^{\mu}\left\{\Phi^{A}, \overline{\mathcal{H}}_{\mu}\right\}, \Phi^{B}+\chi^{\nu}\left\{\Phi^{B}, \overline{\mathcal{H}}_{\nu}\right\}\right\} \\
= & \left\{\Phi^{A}, \Phi^{B}\right\}-\left\{\Phi^{A}, \overline{\mathcal{H}}_{\mu}\right\}\left\{\chi^{\mu}, \chi^{\nu}\right\}\left\{\overline{\mathcal{H}}_{\nu}, \Phi^{B}\right\} \\
& \left.-\left\{\Phi^{A}, \chi^{\mu}\right\}\left\{\overline{\mathcal{H}}_{\mu}, \Phi^{B}\right\}+\left\{\Phi^{A}, \overline{\mathcal{H}}_{\mu}\right\} \chi^{\mu}, \Phi^{B}\right\} \\
& +\mathcal{O}(\chi) \\
= & \left\{\Phi^{A}, \Phi^{B}\right\}^{*}+\mathcal{O}(\chi) .
\end{aligned}
$$

In the last equality we have used the fact that

$$
M=\left(\begin{array}{cc}
\{\overline{\mathcal{H}}, \overline{\mathcal{H}}\} & \{\overline{\mathcal{H}}, \chi\} \\
\{\chi, \overline{\mathcal{H}}\} & \{\chi, \chi\}
\end{array}\right) \approx\left(\begin{array}{cc}
0 & +\delta \\
-\delta & \{\chi, \chi\}
\end{array}\right),
$$

(our fields satisfy the equations of motion, so $\{\overline{\mathcal{H}}, \overline{\mathcal{H}}\} \approx 0^{14}$ ) has as its inverse

$$
M^{-1}=\left(\begin{array}{cc}
\{\chi, \chi\} & -\delta \\
+\delta & 0
\end{array}\right),
$$

thus producing the Dirac brackets above. This computation

\footnotetext{
${ }^{14}$ In Appendix $\mathrm{B}$ we derive the stronger result $\{\overline{\mathcal{H}}, \overline{\mathcal{H}}\}=$ $\mathcal{O}\left(\mathcal{H}^{2}\right)$.
}

has been made at $p$, in the close neighborhood of $p_{G}$. Now we can take the limit $p \rightarrow p_{G}$ on both sides, thus obtaining

$$
\left\{I_{\Phi^{A}},\left.I_{\left.\Phi^{B}\right\}}\right|_{p_{G}}=\left.\left\{\Phi^{A}, \Phi^{B}\right\}^{*}\right|_{p_{G}}=I_{\left.\left\{\Phi^{A}, \Phi^{B}\right\}^{*}\right|_{p_{G}}},\right.
$$

where in the last step we have used the fact that the functionals $I_{\Phi^{A}}$ become the identity functionals when their arguments are taken at $p_{G}$. This concludes the first step of our proof.

An alternative proof, using the connection between the observables and specific canonical transformations of the fields is given in Sec. III C 2.

\section{Step 2: Arbitrary point on gauge orbit}

Let us now extend this result to the entire gauge orbit. We can make an arbitrary gauge transformation sending the equality (37) holding at $p_{G}$ to a corresponding equality at any other point $p$. Let us call $U\left(p, p_{G}\right)$ this gauge transformation from $p_{G}$ to $p$. Its specific descriptors can be determined in a manner similar to the procedure for building the invariants. $U\left(p, p_{G}\right)$ is a canonical transformation and, as such, preserves the Poisson-bracket structure. This means that $U\left(p, p_{G}\right)$ "enters" on both sides of the Poisson bracket. On the other hand, the action of $U\left(p, p_{G}\right)$ on the functionals is

$$
I_{\Phi^{A}}\left[X_{p_{G}}^{\mu}, \Phi_{p_{G}}^{A} ; x\right] \rightarrow I_{\Phi^{A}}\left[X_{p}^{\mu}, \Phi_{p}^{A} ; x\right] .
$$

[In fact the action of $U\left(p, p_{G}\right)$ on these functionals is trivial because they are invariant under the gauge transformations and can be written in terms of the fields at any point in the gauge orbit.] These considerations show that the left hand side of (37) undergoes, under the action of $U\left(p, p_{G}\right)$, the transformation $\left\{I_{\Phi^{A}}, I_{\Phi^{B}}\right\}_{\left.\right|_{p_{G}}} \rightarrow\left\{I_{\Phi^{A}}, I_{\Phi^{B}}\right\}_{\left.\right|_{p}}$.

Let us now address the transformation of the right-hand side of (37) under $U\left(p, p_{G}\right)$. If the canonical gauge transformation $U\left(p, p_{G}\right)$ could "enter" within the Dirac brackets, then the result for the transformation of the right-hand side would be simply $\left\{\Phi^{A}, \Phi^{B}\right\}^{*}{ }_{\left.\right|_{p_{G}}} \rightarrow\left\{\Phi^{A}, \Phi^{B}\right\}_{\left.\right|_{p} ^{*}}^{*}$. But the Dirac bracket structure is not preserved by canonical transformations generated by the Poisson bracket since $\left\{U \Phi^{A}, U \Phi^{B}\right\}^{*} \neq U\left\{\Phi^{A}, \Phi^{B}\right\}^{*}$. Indeed, we can now take advantage of writing $\left.\left\{\Phi^{A}, \Phi^{B}\right\}^{*}\right|_{p_{G}}$ as $I_{\left.\left\{\Phi^{A}, \Phi^{B}\right\}^{*}\right|_{p_{G}}}$ because then $\left\{\Phi^{A}, \Phi^{B}\right\}^{*}$ becomes just the label that identifies the functional we are considering, in the sense that $I_{\left\{\Phi^{A}, \Phi^{B}\right\}^{*}}$ is the functional that sends the specific combination of field configurations given by $\left\{\Phi^{A}, \Phi^{B}\right\}^{*}$ to its value at $p_{G}$. Thus it is obvious that the action of $U\left(p, p_{G}\right)$ on this functional just maps

$$
I_{\left.\left\{\Phi^{A}, \Phi^{B}\right\}^{*}\right|_{p_{G}}} \rightarrow I_{\left.\left\{\Phi^{A}, \Phi^{B}\right\}^{*}\right|_{p}} .
$$

The equality between the transformed objects in the lefthand side and the right-hand side of (37) tells us that we have obtained 


$$
\left\{I_{\Phi^{A}}, I_{\Phi^{B}}\right\} \approx I_{\left\{\Phi^{A}, \Phi^{B}\right\}^{*}}
$$

for any arbitrary point $p$ in the gauge orbit.

\section{Additional remarks}

The results in Sec. II B 3 permit the extension of (38) to the lapse and shift fields. Indeed the situation is the same as in Sec. III B when working with fields other than lapse and shift, but instead of having 4 first-class constraints and 4 gauge-fixing constraints, there are now 8 constraints of each type. Proceeding exactly through the same steps as in Sec. III B, the result (38) can be extended to all the fields, lapse and shift included.

The result (38) has been previously obtained by Thiemann [8] in a remarkable proof based on formal series expansion or-in his own words-by "brutally working out the Poisson brackets." We have provided a natural geometric interpretation of this series expansion.

So far our considerations hold for $\mathcal{S}$, the space of onshell field configurations. But once the results have been obtained, and recalling that all the canonical gauge transformations are active transformations at fixed spacetime coordinates, we can examine all our actions along the gauge orbit at a fixed value of the time coordinate, $t_{0}$. At this fixed time, which can be considered the time for the setting of the initial conditions, the field configurations only need to satisfy the constraints $P_{\mu} \approx 0, \mathcal{H}_{\mu} \approx 0$. Thus our results are valid in a phase-space formulation on the entire original first-class constraint surface (but not including the gauge-fixing constraints, which are only satisfied at the particular point $p_{G}$ in the gauge orbit).

We notice also that the results obtained above are different from the results in [31] showing that the Dirac bracket of the invariant functionals coincides on-shell with its Poisson-bracket; see especially Exercise 1.18 and Sec. 13.2.2 in this book. This is obviously true by the very nature of the invariant functionals; they are required to satisfy $\left\{I_{\Phi^{A}}, G\right\} \approx 0,{ }^{15}$ which is the ingredient needed to show, in the light of the Dirac bracket (36), or its generalization to all $8+8$ constraints, that indeed

$$
\left\{I_{\Phi^{A}}, I_{\Phi^{B}}\right\}^{*} \approx\left\{I_{\Phi^{A}}, I_{\Phi^{B}}\right\}
$$

Taking (38) into account, and including the result above, we can write in phase-space,

$$
\left\{I_{\Phi^{A}}, I_{\left.\Phi^{B}\right\}^{*}} \approx I_{\left\{\Phi^{A}, \Phi^{B}\right\}^{*}},\right.
$$

where we have expressed the fact, using the weak equalities " $\approx$ " that these relations are satisfied on the constraint hypersurface surface in phase space-again, not including the gauge-fixing constraints.

\footnotetext{
${ }^{15}$ At a fixed time $t_{0}$ this reads $\left\{I_{\Phi^{A}}, P_{\mu}\right\} \approx 0,\left\{I_{\Phi^{A}}, \mathcal{H}_{\mu}\right\} \approx 0$.
}

\section{The invariants constructed as limits of canonical maps}

1. On the noncanonicity of the map $\Phi \rightarrow I_{\Phi}$

To further study some aspects of the observables, we will elaborate on the noncanonicity of the map $\Phi \rightarrow I_{\Phi}$ and its proximity to canonical maps. In order not to overload the subsequent considerations we exclude the lapse and the shift fields, but remark that by the techniques described in II B 3 the results of this subsection can be extended to lapse and shift.

Let us recall the expression (16) for the invariant functional $I_{\Phi}$, having chosen the usual basis for the Hamiltonian constraints such that, $\left\{\chi^{\nu}, \overline{\mathcal{H}}_{\mu}\right\}=$ $-\delta_{\mu}^{\nu}+\mathcal{O}(\overline{\mathcal{H}})$ and $\left\{\overline{\mathcal{H}}_{\nu}, \overline{\mathcal{H}}_{\mu}\right\}=\mathcal{O}\left(\overline{\mathcal{H}}^{2}\right)$. Let us write again the expression for our observables, after Eq. (16),

$$
I_{\Phi}:=\exp \left(\left\{-, \bar{\xi}^{\nu} \overline{\mathcal{H}}_{\nu}\right\}\right) \Phi_{\left.\right|_{\xi=\chi}} \approx \sum_{n=0}^{\infty} \frac{1}{n !} \chi^{n}\{\Phi, \overline{\mathcal{H}}\}_{(n)} .
$$

The map $\Phi \rightarrow I_{\Phi}$ sends all the points $p$ in the gauge orbit to a single point $p_{G}$. Hence it can not be a canonical transformation because one such transformation should be invertible. An alternative, indirect but sufficient proof of this noncanonicity is that the Poisson bracket of the invariants associated with two given fields is not the invariant associated with the Poisson bracket of these fields, but with the Dirac bracket. The reason for this noncanonicity may be traced to the fact that the descriptors $\xi^{\nu}$ are replaced by the gauge-fixing constraints $\chi^{\mu}$ after the action of the finite gauge transformation that sends $p$ to $p_{G}$.

We will explore how close this map $\Phi \rightarrow I_{\Phi}$ can be to a canonical transformation. We will show that it is in fact the limit of a family of canonical transformations. To construct this family, an obvious candidate is the object that results from making the replacement of the descriptors before the action of the finite gauge transformation. A one-parameter family of canonical transformations is found by allowing a global rescaling for the descriptors.

So consider the functional, for $G:=\chi^{\nu} \overline{\mathcal{H}}_{\nu}$,

$$
\begin{aligned}
\mathcal{K}_{\Phi} & :=\exp (\{-, \lambda G\}) \Phi=\exp \left(\left\{-, \lambda \chi^{\nu} \overline{\mathcal{H}}_{\nu}\right\}\right) \Phi \\
& =\exp (\{-, \lambda \chi \overline{\mathcal{H}}\}) \Phi,
\end{aligned}
$$

with $\lambda$ a real parameter. Thus the map $\Phi \rightarrow \mathcal{K}_{\Phi}$ is canonical. We will show that $I_{\Phi}$ can be reobtained as the $\lambda \rightarrow \infty$ limit of $\mathcal{K}_{\Phi}$.

We start with the expansion for $\mathcal{K}_{\Phi}$,

$$
\mathcal{K}_{\Phi}=\exp (\{-, \lambda G\}) \Phi=\sum_{n=0}^{\infty} \frac{\lambda^{n}}{n !}\{\Phi, G\}_{(n)} .
$$

To continue, let us define $B_{n}:=\chi^{n}\{\Phi, \overline{\mathcal{H}}\}_{(n)}, n>0$, with $B_{0}:=\Phi$. Our aim is to rewrite the expansion (42) in terms of these objects $B_{n}$. Notice that, due to the fact that 
$\{\chi, \overline{\mathcal{H}}\} \approx-\delta$, we get the simple relation

$$
\left\{B_{n}, G\right\} \approx-n B_{n}+B_{n+1} .
$$

Our result will take the form

$$
\mathcal{K}_{\Phi} \approx \sum_{n=0}^{\infty} c_{n} B_{n},
$$

and the task is to compute the coefficients $c_{n}$.

One can see immediately that $c_{0}=1$. To compute $c_{1}$ we need to add all the appearances of $B_{1}$ in the different terms in (42). We find, keeping only the $B_{1}$ terms,

$$
\begin{aligned}
\mathcal{K}_{\Phi} \approx & \Phi+\lambda B_{1}+\frac{\lambda^{2}}{2 !}\left(-B_{1}+\ldots\right)+\frac{\lambda^{3}}{3 !}\left(B_{1}+\ldots\right) \\
& +\ldots
\end{aligned}
$$

and thus

$$
c_{1}=\lambda-\frac{\lambda^{2}}{2 !}+\frac{\lambda^{3}}{3 !}+\ldots=1-e^{-\lambda} .
$$

It turns out that

$$
c_{n}=\frac{1}{n !}\left(1-e^{-\lambda}\right)^{n}
$$

This result will be obtained below employing a different technique.

With the coefficients (45) we obtain, for (44),

$$
\mathcal{K}_{\Phi} \approx \sum_{n=0}^{\infty} \frac{1}{n !}\left(1-e^{-\lambda}\right)^{n} \chi^{n}\{\Phi, \overline{\mathcal{H}}\}_{(n)} .
$$

It is illuminating to notice the substantial difference between the two series expansions, (42) and (46), for the same functional $\mathcal{K}_{\Phi}$. Both are power series expansions but whereas the first is in terms of the parameter $\lambda$, the second is in terms of $\left(1-e^{-\lambda}\right)$ and is only valid on shell. One reasonably expects convergence at least in the case where the point $p$ in the space of field configurations $\mathcal{S}$ is in the neighborhood of $p_{G}$.

Notice that, as expected, there is no finite $\lambda$ that can make $\mathcal{K}_{\Phi}=I_{\Phi}$. Curiously enough, though, and as long as it is legitimate to enter the limit $\lambda \rightarrow \infty$ within the series expansion (46), one finds, recalling (40),

$$
\lim _{\lambda \rightarrow \infty} \mathcal{K}_{\Phi} \approx I_{\Phi}
$$

thus the invariants can be interpreted as limits of oneparameter families of canonical transformations. But such a limit is no longer a canonical transformation. ${ }^{16}$ Our previous analysis makes the reason more transparent because whereas the limit $\lambda \rightarrow \infty$ can be easily taken for the expansion (46), for which it simply says $\left(1-e^{-\lambda}\right) \rightarrow$

\footnotetext{
${ }^{16}$ Compare with the homothetic map $\mathcal{R}^{2} \rightarrow \mathcal{R}^{2}$ defined by $\vec{v} \rightarrow \frac{1}{\lambda} \vec{v}$, which is invertible for any real value of $\lambda$. It becomes singular for $\lambda \rightarrow \infty: \vec{v} \rightarrow \overrightarrow{0}$.
}

1 , it makes no sense at all for (42). And here is the point: it would have been just by obtaining a finite result for the computation of the limit in the exponent $\{-, \lambda G\}$ in (42), which is clearly divergent, that we would have been assured that the end result was a canonical transformation.

A complementary result is obtained by considering the computation of $\left\{\mathcal{K}_{\Phi}, \overline{\mathcal{H}}\right\}$. It is crucial in this regard that the Poisson bracket of the constraints $\overline{\mathcal{H}}$ among themselves is quadratic in the constraints (see Appendix B). Owing to this fact, $\{G, \overline{\mathcal{H}}\}=-\overline{\mathcal{H}}+\mathcal{O}\left(\overline{\mathcal{H}}^{2}\right)$, and the quadratic terms can be dropped in the internal Poisson brackets as long the final result is expressed on shell. Taking into account (42) and that

$$
\left\{\{\Phi, G\}_{(n)}, \mathcal{H}\right\} \approx \sum_{k=0}^{n}(-1)^{(n-k)} \frac{n !}{k !(n-k) !}\{\{\Phi, \mathcal{H}\}, G\}_{(k)},
$$

one easily obtains ${ }^{17}$

$$
\left\{\mathcal{K}_{\Phi}, \overline{\mathcal{H}}\right\} \approx e^{-\lambda} \mathcal{K}_{\{\Phi, \overline{\mathcal{H}}\}},
$$

which, in the limit $\lambda \rightarrow \infty$, tells us again that $I_{\Phi}$ is an invariant, that is, $\left\{I_{\Phi}, \overline{\mathcal{H}}\right\} \approx 0$.

Notice that we can use (47) to obtain the coefficients (45). Consider the generic expansion (44) for $\mathcal{K}_{\Phi}$ and require that it complies with (47). One easily finds a recurrent equation for the coefficients $c_{n}$,

$$
c_{n}-(n+1) c_{n+1}=e^{-\lambda} c_{n},
$$

which, with the obvious input $c_{0}=1$, yields the result (45)

\section{Revisiting the Dirac bracket}

The considerations in this subsection provide an alternative computation of the Poisson bracket of the invariants as compared to III B. From the definition (41) it is clear that, since $\Phi \rightarrow \mathcal{K}_{\Phi}$ is a canonical transformation,

$$
\left\{\mathcal{K}_{\Phi^{A}}, \mathcal{K}_{\Phi^{B}}\right\}=\mathcal{K}_{\left\{\Phi^{A}, \Phi^{B}\right\}} \text {. }
$$

The fact that (47) implies in general that $\left\{\mathcal{K}_{\Phi}, \overline{\mathcal{H}}\right\} \neq 0$, means that to compute the Poisson bracket $\left\{\mathcal{K}_{\Phi^{A}}, \mathcal{K}_{\Phi^{B}}\right\}$ off-shell information of $\mathcal{K}_{\Phi}$ must be used. The minimal off-shell information we need is $\mathcal{O}(\overline{\mathcal{H}})$. Let us introduce for our purposes the notation $\mathcal{O}(2)$ to describe terms that are quadratic in $\chi^{\mu}, \overline{\mathcal{H}}_{\nu}$. Noticing that for $G:=\chi \overline{\mathcal{H}}$ and arbitrary functionals $\alpha^{\mu}$ and $\beta_{\nu}$, one has

$$
\{\alpha \overline{\mathcal{H}}+\beta \chi, G\}=(\alpha+\beta\{\chi, \chi\}) \overline{\mathcal{H}}-\beta \chi+\mathcal{O}(2),
$$

(where $\beta\{\chi, \chi\} \overline{\mathcal{H}}$ must be interpreted here and in similar

\footnotetext{
${ }^{17}$ This result can also be obtained by differentiating with respect to the parameter $\lambda$ the on-shell equivalent expressions (42) and (46), but here we choose another method, based exclusively on (42), in order to provide a proof of (46).
} 
expressions in the following as $\left.\beta_{\nu}\left\{\chi^{\nu}, \chi^{\mu}\right\} \overline{\mathcal{H}}_{\mu}\right)$ and one easily obtains

$$
\{\Phi, G\}_{(2 n+1)}=\{\Phi, \chi\} \overline{\mathcal{H}}+\{\Phi, \overline{\mathcal{H}}\} \chi+\mathcal{O}(2),
$$

for $n=0,1,2,3, \ldots$, and

$$
\begin{aligned}
\{\Phi, G\}_{(2 n)}= & (\{\Phi, \chi\}+\{\Phi, \overline{\mathcal{H}}\} \chi, \chi\}) \overline{\mathcal{H}}-\{\Phi, \overline{\mathcal{H}}\} \chi \\
& +\mathcal{O}(2),
\end{aligned}
$$

for $n=1,2,3, \ldots$ With these results, using the expansion (41) we find

$$
\begin{aligned}
\mathcal{K}_{\Phi}= & \Phi+\left(1-e^{-\lambda}\right)\{\Phi, \overline{\mathcal{H}}\} \chi+e^{\lambda}\left(\left(1-e^{-\lambda}\right)\{\Phi, \chi\}\right. \\
& \left.\left.+\frac{\left(1-e^{-\lambda}\right)^{2}}{2}\{\Phi, \overline{\mathcal{H}}\} \chi, \chi\right\}\right) \overline{\mathcal{H}}+\mathcal{O}(2) .
\end{aligned}
$$

Note in expression (48) that the off-shell terms diverge for $\lambda \rightarrow \infty$; this means, in particular, that the limit $\mathcal{K}_{\Phi} \rightarrow I_{\Phi}$ for $\lambda \rightarrow \infty$ can only be taken on shell. Let us define, with (46) in mind,

$$
\begin{aligned}
\tilde{\mathcal{K}}_{\Phi} & :=\sum_{n=0}^{\infty} \frac{1}{n !}\left(1-e^{-\lambda}\right)^{n} \chi^{n}\{\Phi, \overline{\mathcal{H}}\}_{(n)} \\
& =\Phi+\left(1-e^{-\lambda}\right)\{\Phi, \overline{\mathcal{H}}\} \chi+\mathcal{O}(2),
\end{aligned}
$$

so finally we have

$$
\tilde{\mathcal{K}}_{\Phi}=\mathcal{K}_{\Phi}-e^{\lambda} \gamma_{\Phi} \overline{\mathcal{H}}+\mathcal{O}(2),
$$

with

$$
\left.\gamma_{\Phi}:=\left(1-e^{-\lambda}\right)\{\Phi, \chi\}+\frac{\left(1-e^{-\lambda}\right)^{2}}{2}\{\Phi, \overline{\mathcal{H}}\} \chi, \chi\right\} .
$$

The functionals $\gamma_{\Phi}$ carry the information of the lowest order off-shell terms for $\mathcal{K}_{\Phi}$. Now we can compute

$$
\begin{aligned}
\left\{\tilde{\mathcal{K}}_{\Phi^{A}}, \tilde{\mathcal{K}}_{\Phi^{B}}\right\}= & \left\{\mathcal{K}_{\Phi^{A}}, \mathcal{K}_{\Phi^{B}}\right\}-e^{\lambda}\left\{\mathcal{K}_{\Phi^{A}}, \overline{\mathcal{H}}\right\} \gamma_{\Phi^{B}} \\
& -e^{\lambda} \gamma_{\Phi^{A}}\left\{\overline{\mathcal{H}}, \mathcal{K}_{\Phi^{B}}\right\}+\mathcal{O}(\overline{\mathcal{H}}, \chi) \\
= & \mathcal{K}_{\left\{\Phi^{A}, \Phi^{B}\right\}}-e^{\lambda}\left\{\mathcal{K}_{\Phi^{A}}, \overline{\mathcal{H}}\right\} \gamma_{\Phi^{B}} \\
& -e^{\lambda} \gamma_{\Phi^{A}}\left\{\overline{\mathcal{H}}, \mathcal{K}_{\Phi^{B}}\right\}+\mathcal{O}(\overline{\mathcal{H}}, \chi),
\end{aligned}
$$

and when we go on shell ( $\overline{\mathcal{H}} \approx 0)$, using (47),

$$
\begin{aligned}
\left\{\tilde{\mathcal{K}}_{\Phi^{A}}, \tilde{\mathcal{K}}_{\Phi^{B}}\right\} \approx & \mathcal{K}_{\left\{\Phi^{A}, \Phi^{B}\right\}}-\mathcal{K}_{\left\{\Phi^{A}, \overline{\mathcal{H}}\right\}} \gamma_{\Phi^{B}}-\gamma_{\Phi^{A}} \mathcal{K}_{\left\{\overline{\mathcal{H}}, \Phi^{B}\right\}} \\
& +\mathcal{O}(\chi) .
\end{aligned}
$$

Next we can take the limit $\lambda \rightarrow \infty$ and we obtain

$$
\begin{aligned}
\left\{I_{\Phi^{A}}, I_{\Phi^{B}}\right\} \approx & I_{\left\{\Phi^{A}, \Phi^{B}\right\}}-I_{\left\{\Phi^{A}, \overline{\mathcal{H}}\right\}}\left(\lim _{\lambda \rightarrow \infty} \gamma_{\Phi^{B}}\right) \\
& -\left(\lim _{\lambda \rightarrow \infty} \gamma_{\Phi^{A}}\right) I_{\left\{\overline{\mathcal{H}}, \Phi^{B}\right\}}+\mathcal{O}(\chi),
\end{aligned}
$$

which explicitly shows the role played by the off-shell terms of $\mathcal{K}_{\Phi}$ in the computation of $\left\{I_{\Phi^{A}}, I_{\Phi^{B}}\right\}$. These terms will gently conspire to bring the Dirac bracket on stage. Indeed, taking the limit $p \rightarrow p_{G}$, which is $\chi \rightarrow 0$, we obtain

$$
\begin{aligned}
\left\{I_{\Phi^{A}}, I_{\left.\Phi^{B}\right\}_{p_{G}}}=\right. & \left\{\Phi^{A}, \Phi^{B}\right\}-\left\{\Phi^{A}, \overline{\mathcal{H}}\right\}\left(\left\{\Phi^{B}, \chi\right\}\right. \\
& \left.\left.+\frac{1}{2}\{\chi, \chi\} \overline{\mathcal{H}}, \Phi^{B}\right\}\right)-\left(\left\{\Phi^{A} \chi\right\}\right. \\
& \left.\left.+\frac{1}{2}\left\{\Phi^{A} \overline{\mathcal{H}}\right\} \chi \chi, \chi\right\}\right)\left\{\overline{\mathcal{H}}, \Phi^{B}\right\} \\
= & \left\{\Phi^{A}, \Phi^{B}\right\}_{\left.\right|_{p_{G}} ^{*}}=I_{\left.\left\{\Phi^{A}, \Phi^{B}\right\}^{*}\right|_{p_{G}}},
\end{aligned}
$$

which is (37).

\section{Extension to all fields including lapse and shift}

Up to this point we have restricted the generic field $\Phi$ on which we operate to be other than the lapse and shift. When $\Phi$ is any generic field, or functional of the fields, the generator of gauge transformations must be taken in its full form (2), or in its equivalent form (24) obtained by the use of a special basis for the constraints, $\bar{\zeta}_{(i) \alpha}=\left(\overline{\mathcal{H}}_{\nu}, \bar{P}_{\mu}\right)$. This is the form that interests us.

By its construction, see Sec. II B 3, this new basis has the property that $\left\{\chi^{(i) \alpha}, \bar{\zeta}_{(j) \beta}\right\} \approx-\delta_{j}^{i} \delta_{\beta}^{\alpha}$, where $\chi^{(i) \alpha}=$ $\left(\chi^{\mu}, \dot{\chi}^{\nu}\right)$ are the secondary and primary gauge-fixing constraints. A bonus of this construction is that the constraints $\bar{\zeta}_{(i) \alpha}$ have strongly vanishing Poisson brackets among themselves. Thus all the properties that have allowed us to obtain results like (37) or (46) hold with the only change being the doubling of the set of constraints involved: Instead of $\overline{\mathcal{H}}_{\nu}$ now we must take $\bar{P}_{\mu}, \overline{\mathcal{H}}_{\nu}$, and instead of $\chi^{\mu}$ now we must take $\dot{\chi}^{\mu}, \chi^{\mu}$. With this simple consideration, all results are extended to any field. In particular the connection between Poisson brackets for the invariants and Dirac brackets-now defined with a set of 16 second class constraints-for the associated fields, and also the obtention of the invariants $I_{\Phi}$ as limits of canonical maps $\mathcal{K}_{\Phi}$, for any field $\Phi$, without restrictions.

\section{CONCLUSIONS}

In 1955 P. G. Bergmann, in a plenary talk in Bern celebrating "Fifty Years of Relativity Theory," expressed the belief that "genuine invariants would reveal themselves as extremely complicated functionals of the presently known field quantities" [35]. Nearly 40 years later Torre confirmed Bergmann's belief with a proof that in generic general relativity no observables exist that can be written as spatial integrals of Cauchy data and finite derivatives thereof [6]. In this paper we have explicitly displayed generic invariants as series involving derivatives of Cauchy data in principle up to infinite order. Others, in particular, Dittrich and Thiemann, have defined invariants as formal power series. However, we are able to establish a relation between functional invariants and specific gauge choices. Equivalently, we have shown that invariants are obtained through a choice of intrinsic coordinates. The 
construction of invariants and the demonstration of the equivalence of the two points of view are achieved through the use of the underlying canonical diffeomorphismsymmetry group of generally covariant theories. We should point out that the observables that are obtained through these constructions are of course not functionally independent. Given the 2 degrees of freedom of pure gravity, there are in phase-space four, or rather $4(\times 3 \times \infty)$ functionally independent observables. The proofs of our results are local in the sense that they can be applied to a region of spacetime. Global issues are not addressed in the present formulation.

We expect that our contribution will help to clarify some controversial issues that are still debated in the literature regarding the notion of gravitational observables. We cite, in particular, the "frozen-time" issue. We identify as a fundamental origin of many of these misunderstandings the fact that different authors do not use the same definition for common words like "gauge transformation.". In Appendix A we have tried, in the guise of an informal dialogue, to pinpoint the most common causes of misunderstanding. We do not, of course, by any means claim to have pronounced the final words on any of them. The fact that our proofs are local means also that we are still far from a final and comprehensive description of the whole picture.

We have established in this paper a broad geometrical interpretation of the construction of observables in generally covariant theories. In particular, we have argued that there exists two basic equivalent points of view as regards the construction of observables once the solutions of the equations of motion are given.

(i) The first point of view, and the one that enjoyed particular emphasis, relies on the existence of a genuine diffeomorphism-induced canonical gauge symmetry group. This group realizes as active canonical transformations all changes of canonical variables that result from general changes of spacetime coordinates. We identify the group as ". diffeomorphism-induced" because the resulting transformations depend on the functional form of some or all of the components of the metric field. Indeed, in order to be able to implement the transformation group, the lapse and shift must be retained as canonical phase-space variables, and permissible diffeomorphisms depend on them in a compulsory manner. We have shown that this group may be employed to construct functions that are invariant under its action. The strategy is to choose an explicit spacetime coordinate-dependent gauge condition, and then to find the finite gauge transformation that transforms the fields to that location on the gauge orbit where the gauge condition is satisfied. The application of this finite transformation to all field variables produces invariants associated with each and every one of them. In order for this program to succeed it is mandatory that the fields ${ }^{18} X^{\mu}$, those that are set equal to the coordinates $x^{\mu}$ in the gaugefixing procedure, transform under general coordinate transformations as spacetime scalars.

(ii) This brings us to the second equivalent view. The gauge choice is nothing other than the selection of that system of spacetime coordinates for which the fields $X^{\mu}$ produce the results $X^{\mu}(x)=x^{\mu}$. This means that we are choosing the values of these scalar fields as the coordinatization of the spacetime. Users sitting at different points $p$ on the gauge orbits have phase-space solutions $\Phi_{p}$ with distinct functional forms. Each is given explicit instructions on developing a potentially infinite series in powers of their coordinates $x^{\mu}$, namely,

$$
\begin{aligned}
I_{\phi} \approx & \sum_{n_{\mu}=0}^{\infty} \frac{1}{n_{0} ! n_{1} ! n_{2} ! n_{3} !}\left(x^{0}\right)^{n_{0}}\left(x^{1}\right)^{n_{1}}\left(x^{2}\right)^{n_{2}} \\
& \times\left(x^{3}\right)^{n_{3}} \mathcal{C}_{n_{0}, n_{1}, n_{2}, n_{3}},
\end{aligned}
$$

with coefficients

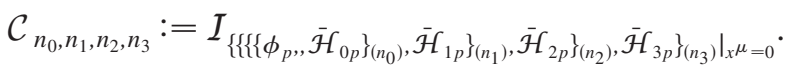

(Where setting $x^{\mu}=0$ refers only to the explicit dependencies.) These coefficients are constant; they are invariant under diffeomorphism-induced canonical transformations, i.e., under displacement from $p$ to $p^{\prime}$ along the gauge orbit. Furthermore, we have shown that these constants are nothing other than the derivatives in a Taylor expansion of solutions at the gauge-fixed location $p_{G}$ on the gauge orbit. Notice that if the user works with the new fields $\hat{\Phi}^{A}=I_{\phi^{A}}$ as the fields of her spacetime, her new description is exactly as if her original coordinates played the role of the intrinsic coordinates.

(iii) We have also proven that the Poisson brackets of the invariants $I_{\phi}$ are identical with the invariant associated with Dirac brackets, i.e., $\left\{I_{\Phi^{A}}, I_{\Phi^{B}}\right\} \approx$ $I_{\left\{\Phi^{A}, \Phi^{B}\right\}^{*}}$. This equality holds for all the canonical variables, including the lapse and shift.

We are able to express all the invariants $I_{\phi}$ as limits of canonical maps applied to the original fields. This aspect throws new light on the emergence of the Dirac bracket just mentioned. On the other hand, out of our invariants, which satisfy the EOM, one can obtain constants of motion with no explicit time dependence. These constants of motion, which are obviously observables, albeit of another kind, are generators of rigid Noether symmetries.

\footnotetext{
${ }^{18}$ These fields may be independent fields or functionals of other fields. An example of the second case is the use by Komar and Bergmann [20] of Weyl scalars, also considered in [10].
} 
(iv) Our results on the Dirac bracket connect our findings with Dirac's procedure for establishing the strong vanishing of Poisson brackets of constraints and gauge conditions through the introduction of Dirac brackets. Of course one must choose gauge conditions of the form $x^{\mu}=X^{\mu}$, where the $X^{\mu}$ are four suitably chosen spacetime scalar functions of the canonical variables. To our knowledge we are the first to establish this detailed connection for the full set of canonical variables. Dirac had originally introduced this method as a way of eliminating inconsistencies in passing from the classical theory to the quantum theory. But since he lacked a geometrical interpretation of the resulting formalism, he and others who followed his lead tended to focus almost exclusively on attempting to identify a minimal complete set of invariants. In particular, the lapse and shift were simply eliminated from the formalism. This was and remains a mistake when passing to the quantum theory. The Dirac bracket of the lapse and shift carries physical information. The quantum noncommutativity of lapse and shift with the remaining quantum observables is an outcome of the specific choice of intrinsic space and time coordinates. As a consequence of this choice the full metric in the quantum theory will be subject to fluctuationyielding a quantum "thickening" of the light cone in an appropriate semiclassical limit.

(v) We think that our approach makes a deep connection with the "evolving constants of motion" program. In particular, the elucidation of the different roles of the explicit and implicit-i.e., through the fields-time dependences in the observables proves to be a key ingredient in the full conceptual clarification of this program.

We wish to stress one additional aspect of our construction of invariants: They are obviously solutions of Einstein's equations, and they make use of a set of selected scalars which define the intrinsic coordinates. It is in this respect that we detect a potential disagreement with the program of partial and complete observables that has been advanced by Rovelli [33], and further elaborated in the canonical framework by Dittrich [7] and Thiemann [8]. Only if the partial variable is a spacetime scalar will their construction of complete observables correspond to an acceptable gauge fixing. In other words, some choices of partial observable as coordinate time might not be legitimate gauge choices.

Many of our findings were conceptually anticipated in Peter Bergmann's short review paper [1]. There we find the idea that, when considered as symmetry generators, the constants of motion obtained from the observables take the configurations out of the gauge orbit. He also anticipated the distinction between the explicit time dependence of the invariants, necessary to enforce the satisfaction of the equations of motion, and the implicit one, which we dis- cuss in Sec. III A 3. Again with the advantage of hindsight one can anticipate from his considerations the "evolving constants of motion" program. He even outlined what should be the role of the Dirac bracket in the algebra of invariants, although without the symmetry group theoretical foundations that we have established in this paper.

\section{ACKNOWLEDGMENTS}

J. M. P acknowledges partial support form MCYT FPA 2007-66665, CIRIT GC 2005SGR-00564, Spanish Consolider-Ingenio 2010 Programme CPAN (CSD200700042). He also thanks the Max Planck Institute for the History of Science and the Theoretical Physics Group at Imperial College London for their warm hospitality at different stages of this work. D. C.S. thanks both Austin College and the Max Planck Institute for the History of Science for their generous support.

\section{APPENDIX A: A DIALOG ON CANONICAL GRAVITY, GAUGE SYMMETRY AND DYNAMICS}

In this appendix we attempt to communicate, in the form of dialogue, our views on some subjects that are still controversial or have been a source of misunderstandings in the canonical formulation of gravity. The interchange is between two subjects, A. and B., the latter representing our point of view. We will essentially touch upon three issues: the gauge group of canonical gravity, the meaning(s) of gauge transformations, and finally the infamous "time is frozen, nothing happens" problem in canonical gravity. We progress from very naïve misunderstandings, which have by now been largely clarified, to some confusions which still persist in the literature. What is said concerning canonical gravity can be extended in obvious ways to other generally covariant theories.

\section{The gauge group of canonical gravity}

A.: Sometimes I ask myself why Einstein's theory, which has such an aesthetic appearance in the Lagrange formulation becomes so ugly looking in its Hamiltonian form. Beyond that, being based upon a $3+1$ decomposition, it is quite clear that canonical gravity is not able to describe the full 4-diffeomorphism invariance of the Lagrangian formulation of general relativity. Having committed to a given $3+1$ decomposition means that a partial gauge fixing is in effect since the diffeomorphisms that do not preserve the foliation must be excluded.

B.: Let us skip matters aesthetic, and cut to the chase. I profoundly disagree with the last thing you said. Nothing prevents diffeomorphisms from acting because the gauge group must be understood as a group of active diffeomorphisms and, as such, it never change the foliation.

A.: But it is clear that a diffeomorphism generated by a vector field $v^{\mu}$ will change the foliation as long as $v^{0}$ depends on the spatial coordinates. 
B.: This is true in the passive view of diffeomorphism invariance, but we are interested in a canonical realization of the gauge group, that is, with generators acting through the Poisson brackets. This is an active action in the sense that it modifies the field configurations but leaves unchanged the coordinates. Active and passive views must be neatly distinguished.

A.: I am happy to concede, but then let me mention what I see as a problem with the canonical realization of the gauge group. Assume that it is possible to treat everything infinitesimally, i.e. near the group identity. We know for instance that in case of an infinitesimal coordinate transformation $\delta x^{\mu}=\hat{x}^{\mu}-x^{\mu}=-\epsilon^{\mu}(x)$ the infinitesimal variation of a tensorial object $T$ is given by the Lie derivative $\mathcal{L}_{\epsilon} T$. (We should leave out further complications due to the presence of spinorial fields in this discussion.) But here things already get hard, since the diffeomorphism group is more complicated than a finite dimensional Lie group. This is, for instance, reflected in the Poisson-bracket structure of the Hamiltonian and momentum constraints in canonical gravity. You do not have structure constants but structure functions. The diffeomorphism group is not realized in phase space.

B.: Not so fast! One must be very careful when moving into phase space. Let me first address some aspects of diffeomorphisms in configuration-velocity space. Bergmann and Komar observed in the early seventies that Einstein's field equations (respectively, the HilbertEinstein action) are not only invariant under point/contact transformations $\left(\hat{x}^{\mu}=f^{\mu}\left(x^{\nu}\right)\right)$, but also under transformations which additionally may depend on the metric fields and their derivatives; i.e. $\left(\hat{x}^{\mu}=f^{\mu}\left(x^{\nu} ; g_{\mu \nu}(x), \ldots\right)\right.$. This is more than a spacetime diffeomorphism in the usual sense of (passive) coordinate transformations. Whereas the diffeomorphism group is acting on the Riemannian manifold (locally describable in the passive view as general coordinate transformations), the larger group, which is certainly a diffeomorphims-induced gauge group, acts on the space of metrics of the Riemannian manifold-and on every other field that is around.

A.: Why make things even more complicated by investigating this far larger group?

B.: The gauge group is what it is, not what you would like it to be. There are many answers to your question, that is, many "becauses": Because an important subgroup of this larger group can be realized in phase space, because this subgroup reveals the explicit form of the gauge generators, because this subgroup gives a clue as to how to interpret even a gravitational Hamiltonian as being responsible for unfolding dynamics, and because this subgroup leads you to a better understanding of observables.

A.: Wow, seems that the "subgroup of the generalized symmetry group" cures my headache-and not only mine. By the way, is this the Bergmann-Komar group?

B.: Well, it depends on who you ask. Some authors mistakenly denote the full metric dependent group as the
Bergmann-Komar group. We reserve this name for the projectable subgroup. Okay, now you can follow me as we explore the fate of symmetries in going from the configuration-velocity space to phase space.

A.: I think we can skip this, since this Dirac procedure is already standard and described in textbooks. At the very end we arrive at a extended Hamiltonian, being the sum of the canonical Hamiltonian and arbitrary linear combinations of the first-class constraints.

B.: Again you are going too fast. Since I know some German: "Soviel Zeit muß sein" - to not forget the contribution of Peter Bergmann and his collaborators (and also that of L. Rosenfeld - but this is another story). But what is more essential for our discussion: We know that because of the singular character of the Lagrangian of general relativity the Legendre transformation from the configurationvelocity space to the phase space is not invertible.

A.: Sorry to interrupt you again, but to arrive at a Hamiltonian even in this singular situation is exactly the task of the Dirac-Bergmann algorithm.

B.: The algorithm is one thing, but understanding the input and the output of the procedure is another story.

A.: The input is the Lagrangian ...

B.: ... with its symmetries. Just wondering-according to you, which generalized diffeomorphism symmetry survives the Legendre transformation?

A.: Is it the Bergmann-Komar group?

B.: You are very clever! Neither the diffeomorphism group nor the larger general field-dependent group allow for a transition from the tangent space to the cotangent space. In order to be Legendre projectable the fielddependent group must be restricted in a specific way, already specified in the 1972 article by Bergmann and Komar.

A.: Could you make this more precise?

B.: Legendre projectability restricts the functional form of the $\epsilon^{\mu}\left(x^{\nu}, g_{\varrho_{\sigma}}, \ldots\right)$ to

$$
\epsilon^{\mu}\left(x^{\nu}, g_{\varrho \sigma}, \ldots\right)=n^{\mu} \xi^{0}+\delta_{a}^{\mu} \xi^{a},
$$

where the $\xi^{\mu}$ are descriptors depending only on the threegeometry (three-metric components and their spatial derivatives) and $n^{\mu}$ is the normal to the $t=$ const hypersurface, expressed by the lapse $N^{0}=N$ and the shift functions $N^{a}$ as

$$
n^{\mu}=\left\{N^{-1},-N^{-1} N^{a}\right\} .
$$

A.: To make things easier let us take the by now standard gauge choice $N=1$ and $N^{a}=0$. We know, and this is already textbook knowledge that "lapse and shift should not be viewed as dynamical variables."

B.: Careful! Through an untimely "so-called" gauge choice you are losing insights in the structure of the phase-space version of gravity: If you fix the lapse and the shift, you are no longer able to identify the "so-called" gauge generators, nor are you able to recognize the differ- 
ence between the so-called gauge generators and the Hamiltonian. And yet you are also worried about the fate of general covariance in going from the Lagrangian to the Hamiltonian in gravitational theories. It turns out, and this is important, that in order to see the diffeomorphisminduced symmetry in phase space one is forced to treat the lapse and the shift functions in the theory as genuine fields.

A.: Okay, I accept that-however with a grain of salt, since I learned that the lapse and the shift functions are devoid of any physical meaning. By the way, why are you so insistent in talking about "so-called" gauge-blah-blah?

B.: I am doing this, because it seems that when considering canonical gravity there seem to be at least two different understandings of what "gauge" means.

A.: Why so? One has the diffeomorhisms...

B.: Which diffeomorphisms do you have in mind here? Automorphic mappings of manifolds, generalized symmetries in the sense of Bergmann and Komar, diffeomorphism-induced transformations in phase space, or perhaps, following Dirac, gauge transformations at a fixed time?

\section{The meaning(s) of gauge transformations}

A.: Okay, you got me. Seems it is time to get deeper into the meaning of gauge symmetries. When Dirac wrote his book, what he had in mind briefly as follows ... wait ... there is a nice description in Rovelli's book [14]: "Consider a system of evolution equations in an evolution parameter $t$. The system is said to be gauge invariant if evolution is under-determined, that is, if there are two solutions that are equal for $t$ less than a certain $t_{0}$."

B.: This is a possible presentation of the "gauge symmetry" phenomenology, but one must proceed with extreme care as regards the definitions. The original diffeomorphism-symmetry maps complete solutions of the field equations to other solutions, but if you read carefully Dirac's book on constrained systems, you will see that when he discusses gauge transformations, he refers to a fixed time, namely $t_{0}$. What makes us believe that Dirac's notion of gauge invariance is the same as the gauge invariance considered by Bergmann, which is the one that maps solutions of the EOM into solutions? I observe that in the community there is no clear distinction among these two notions. And thus there is no clear distinction about what a "gauge generator" is meant to be. This disagreement underlies the famous dispute over whether dynamics is frozen in generally covariant theories like general relativity.

A.: But we know from Dirac's work that all first-class constraints generate gauge transformations.

B. Regretfully Dirac's approach to gauge transformations has caused a lot of misunderstandings. His concept of a gauge transformation was not that of mapping solutions of the EOM into new solutions. He worked at a fixed time - the evolutionary parameter-and so his concept was rather that of relating two sets of initial conditionsat that given time - that, respectively, belong to two gauge equivalent solutions.

A.: So, what is the difference in Dirac's understanding of "gauge transformation" and Bergmann's notion?

B.: That's easy to state: Dirac's "gaugetransformations," generated by all first-class constraints (well, if certain mathematical regularity conditions hold), are valid for a fixed time only-which can be taken as the time at which initial conditions are formulated. But when one considers all possible times, which is necessary if we want to act on a whole solution of the EOM, then the gauge generators, as Bergmann pointed out, are a specific combination of first-class constraints, with a certain number of arbitrary functions and their time derivatives attached to these constraints.

In fact you do not even need to consider general covariance to grasp the distinction between Dirac's and Bergmann's conceptions. Just take pure Maxwell theory with gauge field $A_{\mu}$. There is a primary first-class constraint, namely, the momentum conjugate to $A_{0}$, and a secondary first-class constraint, the Gauss constraint. To generate the gauge transformation $\delta A_{\mu}=\partial_{\mu} \Lambda$ ( $\Lambda$ is an arbitrary function) in phase space, you need to construct a gauge generator made with a specific combination of the two constraints, with coefficients $\Lambda$ for the secondary one and $\dot{\Lambda}$ for the primary one. This is Bergmann's conception. Of course if you consider just a fixed time $t_{0}$, since $\Lambda$ is an arbitrary function, $\Lambda$ and $\dot{\Lambda}$ become independent functions of the spatial coordinates, and that is why in Dirac's view, both constraints generate gauge transformations, but we must insist that this last picture is only valid at a fixed time! You can see with your own eyes that neither of these constraints alone generates transformations mapping solutions into solutions.

In the case of GR, the gauge generators are explicitly

$$
G_{\xi}(t)=P_{\mu} \dot{\xi}^{\mu}+\left(\mathcal{H}_{\mu}+N^{\rho} C_{\mu \rho}^{\nu} P_{\nu}\right) \xi^{\mu} .
$$

Here the $\mathcal{H}_{\mu}$ are the well-known Hamiltonian and momentum constraints, and the $C_{\mu \rho}^{\nu}$ the structure coefficients in their Poisson bracket algebra (called Dirac algebra by some). The $P_{\mu}$ are the momenta canonically conjugate to the lapse and shift functions $N^{\mu}$, and a spatial integration over repeated indices is to be understood. The $\xi^{\mu}$ are arbitrary functions of the spacetime coordinates as well of the field components except for the lapse and shift.

A.: Again I see the lapse and the shift function in this expression. Things would become easier for the gauge choice $N=1, N^{a}=0$.

B.: Yes, things would become easier for some explicit calculations, however not for the interpretation of the gauge generators as generating exactly what they are supposed to generate as symmetry operators, namely, for any object $\phi$ in the theory 


$$
\mathcal{L}_{\xi} \phi=\left\{\phi, G_{\xi}\right\} .
$$

Note that this Poisson bracket is an equal-time bracket. In order to construct the full gauge transformation, mapping solutions into solutions, one needs to consider all times (or at least a finite interval for the time parameter).

\section{Gauge transformations versus dynamical evolution}

A.: Even though it has been hard to follow you with so many different notions of gauge invariance, I think I have finally got you: The Dirac-Hamiltonian for a generally covariant theory is known to be

$$
H_{D}=N^{\mu} \mathcal{H}_{\mu}+\lambda^{\mu} P_{\mu} .
$$

( $\lambda^{\mu}$ are the arbitrary functions of the dynamics.) Thus the choice $\xi^{\mu}=N^{\mu}$ leads to a gauge generator $G_{N}$ once you take into account the equations of motion $\dot{N}^{\mu}=\lambda^{\mu}$. Thus I make the strong claim that the Hamiltonian is a specific gauge generator. And if the Hamiltonian is a gauge generator (even in the sense of Bergmann), how can you escape from interpreting this as leading to "frozentime"? There are no dynamics at all!

B.: Here is the quick and easy response. The generator $\delta t\left(N^{\mu} \mathcal{H}_{\mu}+\dot{N}^{\mu} P_{\mu}\right)$ does serve to replace solutions at time $t$ by the original solutions evaluated at $t-\delta t$. But it performs this function only on one particular member of each equivalence class of solutions, namely, those for which the lapse and shift are the chosen explicit function $N^{\mu}$. On all other members of equivalence classes the effect is to generate variations that are distinct from global translations in time.

But let me try to convince you by looking more closely at the geometry and the transformations we are talking about. For this purpose I will denote the space of fields obeying the GR field equations-one can include matter fields as well-by $\mathcal{S}$; thus points in $\mathcal{S}$ are specific spacetimes with the fields - solutions of the EOM-described in a particular coordinatization. Consider the field content of a point $p$ in $\mathcal{S}$. Let us focus on the data for the fields at time $t_{0}$ and let us call $D$ these data. With a specific selection of the arbitrary functions $\lambda$, there exists a Dirac Hamiltonian, $H(t)=N^{\mu} \mathcal{H}_{\mu}+\lambda^{\mu} P_{\mu}$, which dictates, through the Poisson brackets, the time evolution in $p$. Particularly, for an infinitesimal $\delta t$, this Hamiltonian tells what are to be the field data at the hypersurface labeled by $t_{0}+\delta t$. Let us call these new field data $D^{\prime}$. Now, if we do this for all times $t$, the result is that of course we have remained exactly at the same point $p$ in $\mathcal{S}$, because the dynamics as described by a given observer, takes place within a given spacetime in a given coordinatization.

A.: Is this long exposition meant to persuade me that the Hamiltonian determines the dynamical evolution in phase space?

B.:Well ... yes. But in addition I would like to point out to you the difference between a Hamiltonian and a gauge generator. So let me go on. Consider the gauge generator that, after an appropriate choice of the descriptors, happens to coincide in its mathematical expression with the Dirac Hamiltonian at time $t_{0}$. Because of this coincidence, its action will of course transform the field data $D$ into $D^{\prime}$, but these data $D^{\prime}$ are now conceived at time $t_{0}$, because the gauge transformations are equal-time actions. What happens is that we have moved from $p$ to another gauge equivalent spacetime $p^{\prime}$. If we undertake the same procedure for any time $t$ (continuing to assume that the descriptors at time $t$ match up with the lapse and shift at time $t$ ) we will end up having mapped the whole spacetime $p$ to $p^{\prime}$. Notice that the field configurations in $p$ and $p^{\prime}$ just differ in the time label, and that a passive diffeomorphism $t \rightarrow t-$ $\delta t$ will make both descriptions identical. Obviously this fact should not be a surprise, but should be viewed as a simple consequence of our fundamental understanding of spacetime gauge symmetry. Thus the fact that the gauge generator can mimic the Hamiltonian has nothing to do with the fact that there is real physical ${ }^{19}$ evolution in a given spacetime $p$, where we may consider events, coincidences, causal structure, observables, and so on. Dynamical evolution in $p$ is not gauge action on $p$.

A.: Sorry, you almost manage to confuse me, so let me use my own language. On one side we have $D$ and $D^{\prime}$ as field configurations connected by a gauge transformation. On the other we know that, in some spacetime, the configuration $D^{\prime}$ lies in the future of $D$. Since by definition a gauge transformation does not change the physics, we deduce that the physics in $D$ and $D^{\prime}$ are the same. So the future is gauge equivalent to the past and therefore "nothing happens." How do you address that?

B.: Let me remind you of what we discussed earlier: that in generally covariant theories we must distinguish two notions of gauge transformation, namely, the ones we previously called by the names of Dirac and Bergmann, respectively. Since the symmetry-inspired (Bergmann) notion is about mapping solutions of the equations of motion to solutions, we need to have entire field configurations, not just configurations at a given time $t_{0}$, as occurs with $D$ and $D^{\prime}$. In saying that $D$ and $D^{\prime}$ are connected by a gauge transformation you inadvertently changed the concept of gauge transformation-from Bergmann's to Dirac's—but intended to keep intact its interpretation. That is a mistake.

A.: Just to get your point: Are you saying that the phrase "A gauge transformation does not change the physics" is wrong?

B.: It depends on what you mean by "the physics" and by a "gauge transformation." Note that $D$ and $D^{\prime}$ can be conceived as settings of initial conditions. The fact that these two sets of initial conditions are related by a gauge transformation effected at a given time $t_{0}$ means that both $D$ and $D^{\prime}$ are good data to build, using the dynamics, the

\footnotetext{
${ }^{19}$ See below for clarifications on the meaning of "physics."
} 
same physics. This is what we have seen before with the spacetimes $p$ and $p^{\prime}$, which are gauge equivalent. With the word "physics" here we mean the entire spacetime, with the entire history, modulo gauge transformations. We may call this physics the "entire physics". This is the physics that enters in your sentence "a gauge transformation does not change the physics," because it refers to mapping solutions into solutions. In this physics the statement is true.

A.: This being said, I assume that there is another meaning of physics in which the sentence, "A gauge transformation does not change the physics," is indeed wrong.

B.: Yes indeed. But you must take now Dirac's gauge transformations at a single time. As we have just seen, $D$ and $D^{\prime}$ are related in this sense. It is obvious that if $D^{\prime}$ is a fixed-time field configuration in the future of $D$ (we may consider here finite time separations instead of infinitesimal ones) in a given spacetime $p$, both are equally good data from which one can reconstruct the entire spacetime and so both belong to the same "entire-physics". But if we prefer to stay in a more down-to-earth perspective, regarding configurations at a given time (let us call it "timeslicephysics") in a given spacetime, then of course the timeslice-physics in $D$ and in $D^{\prime}$ can be very different, although the entire physics is the same. Perhaps in $D$ you were not born yet and in $D^{\prime}$ you were. That is a big difference, and observable, is it not?

\section{APPENDIX B: FROM WEAKLY TO STRONGLY VANISHING POISSON BRACKETS OF FIRST- CLASS CONSTRAINTS}

In their monograph [31] Henneaux and Teitelboim considered in chapter 5.2 the idea of "Abelianization of constraints," an idea already present in another language in classical monographs on differential equations, like [36]. In this respect, a particularly efficient technique is that of Dittrich [7] and Thiemann [8], which we adopt in II B 1. We will prove that these "Abelianized" constraints lead to strongly vanishing first-class constraints in the sense of Dirac. In Dirac's terminology, a function $f$ strongly vanishes (denoted as $f \equiv 0$ ) in phase space if it vanishes and in addition its differential also vanishes on the constraint surface.

For simplicity, we shall use the language of mechanics. Consider a $d$-dimensional manifold with $n\left(n \leq \frac{d}{2}\right)$ firstclass, independent, and effective constraints $\phi_{i}$. (A constraint is said to be effective if it has a non vanishing differential on the constraint's surface.) They define the surface $\mathcal{M}$ and so they satisfy $\left\{\phi_{i}, \phi_{j}\right\}=f_{i j}^{k} \phi_{k}$.
Associated vector fields are

$$
V_{i}=\left\{-, \phi_{i}\right\}
$$

so that

$$
\left[V_{i}, V_{j}\right] \approx f_{i j}^{k} V_{k},
$$

where the symbol $\approx$ means that the equality is valid on $\mathcal{M}$.

Now consider $n$ independent functions $F^{i}$ such that $\operatorname{det} V_{i}\left(F^{j}\right) \neq 0$. Next take the functions $F^{i}, \phi_{j}$, and $d-$ $2 n$ extra functions to make a change of coordinates in the phase space, at least in a neighborhood of $\mathcal{M}$. If the original coordinates-positions and momenta-where $x$, we call the new coordinates $y$ so that $y^{i}=F^{i}(x), y^{a}=$ $F^{a}(x)$, where $a=n+1 \ldots d$ and $F^{a}$ include the constraints $\phi_{i}$.

Express the vector fields in the new coordinates

$$
V_{i}=V_{i}\left(F^{j}\right) \partial_{y^{j}}+V_{i}\left(F^{a}\right) \partial_{y^{a}} .
$$

Next define $B_{i}^{j}=\left(V_{i}\left(F^{j}\right)\right)^{-1}$ and make independent linear combinations of the vector fields by defining $\bar{V}_{i}=B_{i}^{j} V_{j}$. It turns out that

$$
\bar{V}_{i}=\partial_{y^{i}}+D_{i}^{a} \partial_{y^{a}},
$$

for some coefficients $D_{i}^{a}$. Since we just made a linear combination of the vector fields, they still satisfy a closure property on $\mathcal{M}$,

$$
\left[\bar{V}_{i}, \bar{V}_{j}\right] \approx \bar{f}_{i j}^{k} \bar{V}_{k},
$$

but on the other hand, given the form of $\bar{V}_{i}$ in the new coordinates, it is clear that $\left[\bar{V}_{i}, \bar{V}_{j}\right]$ can not have $\partial_{y^{k}}$ terms on the right-hand side and therefore we can not write $\bar{V}_{k}$ on the right-hand side. We conclude that $\left[\bar{V}_{i}, \bar{V}_{j}\right] \approx 0$ (or equivalently, $\bar{f}_{i j}^{k} \approx 0$ ).

Now consider a change of basis for the constraints, along the same lines, that is, $\bar{\phi}_{i}=B_{i}^{j} \phi_{j}$., then

$$
\left\{-, \bar{\phi}_{i}\right\}=\bar{V}_{i}+\mathcal{O}(\phi),
$$

where by $\mathcal{O}(\phi)$ we mean vector fields that vanish on $\mathcal{M}$. We thus have

$$
\begin{aligned}
\left\{f,\left\{\bar{\phi}_{i}, \bar{\phi}_{j}\right\}\right\} & \approx\left[\bar{V}_{i}+\mathcal{O}(\phi), \bar{V}_{j}+\mathcal{O}(\phi)\right] f \approx\left[\bar{V}_{i}, \bar{V}_{j}\right] f \\
& \approx 0,
\end{aligned}
$$

for any function $f$, which means that

$$
\left\{\bar{\phi}_{i}, \bar{\phi}_{j}\right\}=\mathcal{O}\left(\phi^{2}\right) \equiv 0 .
$$


[1] P. G. Bergmann, Rev. Mod. Phys. 33, 510 (1961).

[2] L. Lusanna and M. Pauri, Gen. Relativ. Gravit. 38, 187 (2006).

[3] D.C. Salisbury, in The Eleventh Marcel Grossmann Meeting On Recent Developments in Theoretical and Experimental General Relativity, Gravitation and Relativistic Field Theories, edited by H. Kleinert, R. Jantzen, and R. Ruffini (World Scientific, New Jersey 2008), p. 2467.

[4] D. C. Salisbury, arXiv:0904.3993.

[5] T. Regge and C. Teitelboim, Ann. Phys. (N.Y.) 88, 286 (1974).

[6] C. G. Torre, Phys. Rev. D 48, R2373 (1993).

[7] B. Dittrich, Gen. Relativ. Gravit. 39, 1891 (2007).

[8] T. Thiemann, Classical Quantum Gravity 23, 1163 (2006).

[9] J. M. Pons, D. C. Salisbury, and L. C. Shepley, Phys. Rev. D 55, 658 (1997).

[10] J. M. Pons and D. C. Salisbury, Phys. Rev. D 71, 124012 (2005).

[11] D. C. Salisbury, J. Helpert, and A. Schmitz, Gen. Relativ. Gravit. 40, 1475 (2008).

[12] J. M. Pons, D. C. Salisbury, and K. A. Sundermeyer, Mod. Phys. Lett. A 24, 725 (2009).

[13] R. M. Wald, General Relativity (University Press, Chicago 1984), p. 473.

[14] C. Rovelli, Quantum Gravity (Cambridge Univ. Pr., Cambridge, 2004), p. 455.

[15] C. Rovelli, in Conceptual Problems in Quantum Gravity, edited by A. Ashtekar and J. Stachel (Birkhauser, Boston, 1991), p. 126.

[16] C. Rovelli, Phys. Rev. D 43, 442 (1991).

[17] C. Rovelli, Phys. Rev. D 42, 2638 (1990).

[18] P. G. Bergmann and A. Komar, Int. J. Theor. Phys. 5, 15 (1972).
[19] C. Rovelli, Phys. Rev. D 65, 044017 (2002).

[20] P. G. Bergmann and A. Komar, Phys. Rev. Lett. 4, 432 (1960).

[21] J. Stachel, in Einstein and the History of General Relativity, edited by D. Howard and J. Stachel (Birkhauser, Boston, 1989).

[22] B. DeWitt, in Gravitation; an Introduction to Current Research, edited by L. Witten (Wiley, New York, 1962).

[23] C. Isham and K. Kuchar, Ann. Phys. (N.Y.) 164, 288 (1985).

[24] J. Hartle, in Conceptual Problems of Quantum Gravity, edited by A. Asthekar and J. Stachel (Birkhauser, New York, 1991).

[25] L. Lusanna, arXiv:gr-qc/0302089.

[26] J. D. Brown, K. V. Kuchar, Phys. Rev. D 51, 5600 (1995).

[27] K. Sundermeyer, Lect. Notes Phys. 1691 (1982).

[28] J. M. Pons and L. C. Shepley, Classical Quantum Gravity 12, 1771 (1995).

[29] P. A. M. Dirac, Lectures on Quantum Mechanics (Yeshiva University Press, New York, 1964).

[30] J. M. Pons, Stud. Hist. Philos. Mod. Phys. 36, 491 (2005).

[31] M. Henneaux and C. Teitelboim, The Quantization of Gauge Systems (Princeton University Press, Princeton, 1994).

[32] A. Anderson, arXiv:gr-qc/9507038.

[33] C. Rovelli, Phys. Rev. D 65, 124013 (2002).

[34] C. Batlle, J. Gomis, X. Gracia, and J. M. Pons, J. Math. Phys. (N.Y.) 30, 1345 (1989).

[35] P. G. Bergmann, Helv. Phys. Acta Suppl. IV, 90: "Wahre Invarianten, glaube ich, werden sich als äußerst komplizierte Funktionale der gegenwärtig bekannten Feldgrößen entpuppen."

[36] L.P. Eisenhart, Continuous Groups of Transformations (Dover Publications, Inc., New York, 1961). 